TIFR/TH/12-38

$\mathrm{HRI} / \mathrm{ST} / 1210$

ICTS/2012/11

\title{
CMB From CFT
}

\author{
Ishan Mata, ${ }^{1}$ Suvrat Raju $\mathbf{u}^{2,3,4}$ and Sandip P. Trivedi ${ }^{1}$ \\ ${ }^{1}$ Tata Institute Of Fundamental Research, 1 Homi Bhabha Road, Colaba, Mumbai 400 005, India. \\ ${ }^{2}$ International Centre for Theoretical Sciences, TIFR, IISc Campus, Bangalore 560012, India. \\ ${ }^{3}$ Harish-Chandra Research Institute, Jhunsi, Allahabad 211019, India. \\ ${ }^{4}$ School of Natural Sciences, Institute for Advanced Study, Princeton, NJ 08540, USA. \\ E-mail: ishanmata@gmail.com, suvrat@icts.res.in, trivedi.sp@gmail.com
}

\begin{abstract}
During inflation, spacetime is approximately described by de Sitter space which is conformally invariant with the symmetry group $S O(1,4)$. This symmetry can significantly constrain the quantum perturbations which arise in the inflationary epoch. We consider a general situation of single field inflation and show that the three point function involving two scalar modes and one tensor mode is uniquely determined, up to small corrections, by the conformal symmetries. Special conformal transformations play an important role in our analysis. Our result applies only to models where the inflaton sector also approximately preserves the full conformal group and shows that this three point function is a good way to test if special conformal invariance was preserved during inflation.
\end{abstract}

KEYworDS: inflation, correlation functions, de Sitter space, conformal field theory 


\section{Contents}

1 Introduction 1

2 Basic Set-Up $\quad 5$

3 Constraints of Conformal Invariance on the Correlation Function 15

4 Solving the Conformal Constraints $\quad 23$

5 Final Result $\quad 31$

6 Conclusions $\quad 31$

A The Two Point Function and Normalizations 34

B Details of the Equations for Special Conformal Invariance 35

$\begin{array}{ll}\text { C Spinor Helicity Formalism } & 37\end{array}$

\section{Introduction}

Inflation states that our Universe underwent a period of exponentially rapid expansion in its early history. This idea solves the flatness and horizon problems in cosmology. What is particularly attractive is that the same exponential expansion also results in small quantum perturbations being produced which account for the observed anisotropies of the microwave background and also provide the seed perturbations for the growth of large scale structure in the Universe.

The exponentially expanding Universe during inflation is well described by the metric of de Sitter space, up to small corrections. It is well known that de Sitter space is a maximally symmetric spacetime. In four dimensions the group of isometries of de Sitter space is $S O(1,4)$ - the Lorentz group in $4+1$ dimensional flat spacetime. This large group of symmetries has ten generators, which include translations and rotations along the three space directions, scale transformations, and the three generators of special conformal transformations. We will refer to it as the conformal group below.

So far, the experimental tests of inflation, coming for example from the study of the $\mathrm{CMB}$, have shown that the perturbations can be well approximated as being Gaussian. The good news is that future experiments, with improved sensitivity, will be able to probe and possibly detect evidence for non-Gaussianity in these perturbations. For example, it is hoped 
that the Planck experiment will be able to provide significant constraints of this sort quite soon.

A Gaussian distribution is completely determined by its two point correlation function. Any non-Gaussianity in the perturbations can therefore be characterized by the three point or higher point correlations. Considerable attention has been paid in the recent literature to the three-point function, called the bispectrum; there is also a growing body of literature on the four point function, called the trispectrum. We refer the reader to $[1,2]$ for a review of these developments and to [3] for background material.

There are two kinds of perturbations of the metric that are relevant for inflation: these transform as scalars and spin-2 representations of the rotation group, and are called scalar and tensor perturbations respectively. In addition each perturbation is characterized by a value for the spatial three-momentum. It is easy to see that the momentum dependence of the two-point function of the perturbations is simple and is fixed, up to small corrections, by the approximate scale invariance of de Sitter space. One the other hand, it is well known that the momentum dependence of the three point functions can be much more complicated. For example, various different shapes which characterize this momentum dependence have been obtained for the three point scalar correlation function in different models of inflation. (See $[1,4]$ and references there.)

The symmetries of de Sitter space need not be shared by the scalar sector in general. This happens for example in DBI inflation [5] where the non-canonical kinetic energy term for the inflaton results in a speed of sound $c_{s} \neq 1 .{ }^{1}$ As a result, while scaling symmetry is preserved, the inflaton sector breaks special conformal invariance badly. Here we will assume that the full conformal group is approximately preserved by the inflationary dynamics, including both gravity and the inflaton field, and examine the resulting constraints imposed on three point functions.

In particular, we will focus on the three point function involving two scalar perturbations $\zeta(\boldsymbol{k})$ and one tensor perturbation $\gamma_{i j}(\boldsymbol{k})$, with polarization $e^{s, i j}$, denoted by,

$$
\left\langle\zeta\left(\boldsymbol{k}_{1}\right) \zeta\left(\boldsymbol{k}_{2}\right) \gamma_{i j}\left(\boldsymbol{k}_{3}\right)\right\rangle e^{s, i j}
$$

We will show that this correlator is completely fixed by symmetry considerations. ${ }^{2}$ Its overall normalization is determined in terms of the two point functions of the scalar and tensor perturbations, and its momentum dependence is determined by the $S O(1,4)$ symmetry group. It turns out that the special conformal transformations play an especially important role in our analysis. They give rise to differential equations for the correlation function whose solution is essentially unique leading to the conclusion above. In the absence of special conformal invariance in the full theory, including the inflaton sector, our results for the correlator are not valid.

\footnotetext{
${ }^{1}$ Another example where the scalar sector violates special conformal symmetries is ghost inflation [6].

${ }^{2} \mathrm{~A}$ complete complete definition of the perturbations etc. is given in section 2 .
} 
Our analysis applies to models with only one scalar field during inflation. It also assumes that the initial state was the Bunch-Davies vacuum. ${ }^{3}$ Beyond that, our analysis only relies on the conformal group and is essentially model independent. In particular, our results also apply to models where higher derivative corrections are important and gravity or the scalar field is not well described by the two-derivative approximation. In the context of string theory, such a situation would arise if the Hubble scale $H$ during inflation was of order the string scale $M_{s t}$. Present bounds on $H$ coming, for example, from the absence of any observed effects due to tensor perturbations tell us that $H<\sim 10^{16} \mathrm{Gev}<M_{P l}$. So, for example, the higher derivative corrections would be important if $H$ and $M_{\text {st }}$ are both comparable and of order the Grand unification scale $M_{\mathrm{GUT}} \sim 10^{16} \mathrm{Gev}$. Since very little is understood about string theory in time dependent backgrounds the resulting correlation functions in such a situation cannot be calculated directly from our present knowledge of the theory. However symmetry considerations still hold and our result for the correlation function (1.1) is valid for such a situation as well.

The generality of our result makes the correlator given in (1.1) a good test, in a model independent manner, of the full symmetry group during inflation. The two-point scalar correlator, which has now been measured, is consistent with approximate scale invariance but this leaves open the possibility that the special conformal symmetries of de Sitter space are not preserved by the scalar sector. In fact, as was mentioned above, it is easy enough to construct models of inflation where this does happen and also straightforward to see that this possibility is allowed in terms of an effective field theory analysis [7]. The correlator discussed here, if observationally measured, can conclusively settle whether the special conformal symmetries were approximately preserved during inflation.

Unfortunately, experimental tests of this three point correlator are still some way away since its magnitude is small. Even the detection of the two point function for the tensor mode has not been made so far and would be a great discovery in itself. The small value that the three point scalar correlator has in conventional slow-roll inflation can be enhanced in models like DBI inflation which involve the breaking of special conformal symmetries. However, with the special conformal symmetries intact our analysis fixes the the overall normalization of the correlation function with two scalars and one tensor, as was mentioned above, and rules out the possibility of any such enhancement.

Therefore, we present the result of our analysis here not with any immediate experimental contact in mind, but rather with a view to the future when hopefully such contact will become possible and such model independent tests of inflation might play a useful role in sharping our understanding of the early Universe.

A second motivation for our work comes from the study of conformal field theory. The symmetry group mentioned above, $S O(4,1)$, is exactly the same as the symmetry group of a 3 dimensional Euclidean conformal field theory (CFT). This is in fact why we referred to this symmetry group as the conformal group when we first introduced it above. The problem

\footnotetext{
${ }^{3}$ These boundary conditions are restated in a way more convenient for our analysis in section 2.3.
} 
of studying the constraints imposed by this symmetry group on the correlation functions of the scalar and tensor perturbations in de Sitter space maps in a direct way to the question of studying the constraints imposed in a 3 dimensional conformal field theory on correlation functions involving a nearly marginal scalar operator and the stress energy tensor. Thus our analysis is also of interest in the study of 3 dimensional CFTs: a subject which has also been of some considerable interest recently. ${ }^{4}$

The three point correlation function for two scalar operators and the stress tensor is already well known in the CFT literature [8]. However this result is in position space, while for cosmology one is interested in the answer in momentum space. It is not easy to directly Fourier transform the position space result. Moreover, the position space answer has divergences where the operators come together. It is rather subtle to regulate these divergences - which is necessary to define the Fourier transform - while preserving conformal invariance. A closely related issue is that of contact terms, which can also arise in position space. These were not determined in [8] but are important for the momentum dependence of the correlator. As our analysis shows, working directly in momentum space, the symmetry considerations are powerful enough to fix these ambiguities for the correlator and determine a unique answer.

Finally, a third motivation comes from attempts to study de Sitter space and its possible dual description in terms of a CFT $[10,11,12]$. It is unclear at this point whether a precise correspondence of this type is possible. However, symmetry properties for correlators can be related between the gravity description and the CFT, as mentioned above. These are analogous to and in fact follow after analytic continuation from the correspondence between correlators in the AdS/CFT case. Since, as our results help show, symmetry properties can significantly constrain at least some of the correlators, the correspondence in this limited sense is still of some practical benefit.

Before going further we must mention the seminal papers of Maldacena [11] and more recently Maldacena and Pimentel [13]. These papers lay out the essential ideas on which our analysis is based. The precise nature of the map between the gravity theory and the CFT using the wave function of the Universe was first discussed in [11]. And the importance of special conformal transformations was discussed in [13] where it was also shown that these symmetries significantly constrain the three point function of tensor perturbations. Our analysis is a modest extension of this approach for a correlator involving scalar perturbations as well.

Other relevant works which explore similar ideas are [14, 7, 15]. Two recent papers [16] appeared while this paper was being prepared for publication and contain related material.

This paper is organized as follows. In $\S 2$ we discuss the basic ideas behind the analysis and background material. In $\S 3$ we set up the equations which arise due to conformal invariance. In $\S 4$ we discuss a solution to these equations and prove that it is unique. Our final results are presented in $\S 5$. We end with conclusions in $\S 6$. Three Appendices contain important supplementary material follow. A reader who is not interested in the details of the calculations

\footnotetext{
${ }^{4}$ For some discussion of three-point functions in 3 dimensional CFTs see [8, 9].
} 
can read the introduction, and then turn directly to $\S 5$ with the final results, which can be read in a self contained way together with Appendix A, and then end with the conclusions.

\section{Basic Set-Up}

We consider a theory of gravity coupled to a scalar field, the inflaton, with action

$$
S=\int d^{4} x \sqrt{-g} \frac{1}{16 \pi G}\left[R-\frac{1}{2}(\nabla \phi)^{2}-V(\phi)+\cdots\right] .
$$

The ellipses stand for higher derivative corrections involving, in general, both gravity and the inflaton. Such corrections could be important, for example, if the Hubble scale during inflation is of order the string scale. Note that in (2.1) we are using conventions where the inflaton is dimensionless. Also below we will choose conventions where the Planck scale

$$
M_{P l}^{2} \equiv 8 \pi G=1 .
$$

It is well known that during inflation the Universe is approximately described by de Sitter space

$$
\begin{aligned}
& d s^{2}=-d t^{2}+a^{2}(t) \sum_{i=1}^{3} d x_{i} d x^{i}, \\
& a^{2}=e^{2 H t}
\end{aligned}
$$

and hence undergoes exponential expansion. In (2.4), $H$ is the Hubble scale which is a constant in de Sitter space. The inflationary epoch is described by de Sitter space with small corrections. These arise because of the slow variation of the Hubble scale which can be parametrized in terms of the two parameters

$$
\epsilon=-\frac{\dot{H}}{H^{2}}, \delta=\frac{\ddot{H}}{2 H \dot{H}},
$$

where dot denotes derivative with respect to $t$. During inflation both these parameters are small and meet the slow roll conditions

$$
\epsilon, \delta \ll 1 \text {. }
$$

When the two-derivative approximation is good and the action can be approximated by the terms given in (2.1), $H$ is given in terms of $V$ by

$$
H=\sqrt{\frac{V}{3 M_{P l}^{2}}},
$$

and the slow roll parameters can be expressed in terms of of $V$ by

$$
\begin{aligned}
& \epsilon=\frac{1}{2} \frac{M_{p l}^{2}\left(V^{\prime}\right)^{2}}{V^{2}}, \\
& \delta=-M_{p l}^{2} \frac{V^{\prime \prime}}{V}+\epsilon,
\end{aligned}
$$


where prime denotes derivatives with respect to the scalar field. ${ }^{5}$ Also in the two-derivative theory we have

$$
\epsilon=\frac{1}{2} \frac{\dot{\phi}^{2}}{H^{2}}
$$

When the two-derivative approximation is not valid $\epsilon$ defined in (2.5) and $\dot{\phi}$ will not be related by (2.10) in general. The slow-roll approximation then requires that besides (2.6) being valid,

$$
\frac{\dot{\phi}}{H} \ll 1
$$

de Sitter space is well known to be conformally invariant. For example it is easy to see that the scale transformation

$$
x^{i} \rightarrow \lambda x^{i}, t \rightarrow t-\frac{1}{H} \log (\lambda),
$$

leaves the metric (2.3) invariant. More generally the full isometry group of de Sitter space is $S O(1,4)$. It consists of the usual three translations and rotations in the $x^{i}$ coordinates, the scale transformation, (2.6), and in addition three special conformal transformations. Infinitesimal special conformal transformations are of the form

$$
\begin{aligned}
& x^{i} \rightarrow x^{i}-2\left(b_{j} x^{j}\right) x^{i}+b^{i}\left(\sum_{j}\left(x^{j}\right)^{2}-e^{-2 H t}\right), \\
& t \rightarrow t+2 b_{j} x^{j}
\end{aligned}
$$

Here $b^{i}, i=1, \ldots 3$ are infinitesimal parameters. As mentioned above de Sitter space is modified during inflation due to the time varying Hubble scale. While translations and rotations in the $x^{i}$ directions are of course unbroken, this modification results in the breaking of the scaling and special conformal symmetries. However, as long as the slow roll parameters $\epsilon, \delta$, are small this breaking is small and the resulting inflationary spacetime is still approximately conformally invariant.

The inflaton sector need not preserve the full conformal group breaking the $S O(1,4)$ symmetry of de Sitter space badly and only preserving translations, rotations and scale transformations, as was mentioned in the introduction. Additional parameters enter in such a model which parameterize this breaking. For example, the speed of sound, $c_{s}$, is one such parameter. When $c_{s} \neq 1$ the special conformal symmetries are broken. See [7] for a more general parametrization of such effects. In what follows we will assume that the scalar sector also approximately preserves the full symmetry group of de Sitter space.

\subsection{The Perturbations}

The inflationary space-time is a solution for the system consisting of gravity and a scalar field. The rotational invariance in the $x^{i}$ directions can be used to characterize perturbations

\footnotetext{
${ }^{5}$ The slow-roll parameter $\eta$ which is more conventionally used is given by $\eta=M_{P l}^{2} \frac{V^{\prime \prime}}{V}$.
} 
about this solution. There are two kinds of perturbations which can arise, scalar and tensor perturbations. The scalar perturbations have spin zero and the tensor perturbations have spin 2.

The tensor perturbations are easy to understand - they are gravity waves in the inflationary background. The scalar perturbations essentially arise due to the presence of the inflaton field. Depending on the gauge chosen they can be thought of as perturbations in the inflaton, or in the spatial curvature or in a combination of both of these modes.

\subsubsection{Gauge 1}

For example, we can choose a gauge where the perturbations in the inflaton vanish,

$$
\delta \phi=0
$$

Starting with the form of the metric used in the ADM formalism

$$
d s^{2}=-N^{2} d t^{2}+h_{i j}\left(d x^{i}+N^{i} d t\right)\left(d x^{j}+N^{j} d t\right)
$$

the additional coordinate reparameterization can be fixed by choosing a gauge where

$$
h_{i j}=a^{2}\left[(1+2 \zeta) \delta_{i j}+\gamma_{i j}\right]
$$

where $\gamma_{i j}$ is transverse and traceless,

$$
\partial_{i} \gamma_{i j}=\gamma_{i i}=0
$$

as discussed in [11]. The tensor perturbations are given by $\gamma_{i j}$. And the scalar perturbations are given by $\zeta$ and correspond to fluctuations in the spatial curvature along the spatial directions.

\subsubsection{Gauge 2}

Alternatively, for the scalar perturbations, we can choose to set $\zeta$ instead of $\delta \phi$ to vanish. The perturbations are now given by fluctuations in the inflaton, $\delta \phi$. This second gauge is obtained by starting with the coordinates in which the perturbations take the form given in the previous paragraph, $\zeta, \gamma_{i j}$ and carrying out a time reparameterization

$$
t \rightarrow t+\frac{\zeta}{H}
$$

It is easy to see that this sets $\zeta$ to vanish. The tensor perturbation $\gamma_{i j}$ is unchanged by this coordinate transformation. If the background value of the inflaton in the inflationary solution is

$$
\phi=\bar{\phi}(t)
$$

the resulting value for the perturbation $\delta \phi$ this gives rise to is

$$
\delta \phi=-\frac{\dot{\bar{\phi}} \zeta}{H} .
$$


When the two derivative approximation is good we can using (2.10) express this relation as

$$
\delta \phi=-\sqrt{2 \epsilon} \zeta .
$$

We will find it useful to consider both gauges in our discussion below. As we will discuss further in subsection 2.3 for our purposes it will be most convenient to first work in gauge 2 , where the scalar perturbation is given by $\delta \phi$ and then transform to gauge 1 , where the perturbation is given by $\zeta$, around the time when the mode crosses the horizon. This might seem conceptually confusing at first but has the advantage of allowing us to incorporate both the leading effects of the slow-roll parameters in a straightforward manner and of eventually going over to the description in terms of $\zeta$ which is the variable that it is defined for all time and also becomes constant once the mode exits the horizon.

Let us also make one more comment here. The relation (2.21) has corrections involving higher powers of the perturbation, $\delta \phi$. For the scalar three-point function in conventional slow-roll models, as studied in [11], the first corrections to (2.21) need to be kept since the leading answer is suppressed by an additional power of $\sqrt{\epsilon}$. But these corrections can be ignored for the correlator (1.1).

\subsection{The Wave Function}

The time dependence during the inflationary epoch gives rise to scalar and tensor perturbations. Our main interest in this paper is to ask about the constraints that approximate conformal invariance imposes on the correlation functions of these perturbations. In particular we will be interested in these correlation functions at late enough times when the modes have crossed the horizon, and their wavelength, $\lambda$, has become much bigger than the Hubble scale, $\lambda \gg H^{-1}$.

At such late times the correlations functions acquire a time independent limiting form. The physical reason for this is well understood. Once the wavelength of a mode gets much longer than the Hubble scale the evolution of the mode gets dominated by Hubble friction and as a result it comes to rest.

In our discussion it will be useful to think in terms of a wavefunction which describes the state of the system at late times. The wavefunction tells us the amplitude to observe a particular perturbation and clearly encodes all information about the correlation functions. Since the correlation functions become time independent at late times the wave function also becomes time independent in this limit. ${ }^{6}$

The wave function will be a convenient description for our analysis since we are interested in the constraints imposed by symmetries and these can be conveniently translated to invariances of the wavefunction as we will see shortly. In turn this will allow us to map the constraints imposed by symmetries to an analysis of constraints imposed on correlators in a 3 dimensional Euclidean conformal field theory. More generally, thinking in terms of the wave function also allows us to exploit the analogy with calculations in AdS space for our purpose.

\footnotetext{
${ }^{6}$ More accurately, this happens after suitable infra-red divergences are subtracted. Physical answers do not depend on the choice of subtraction procedure.
} 
The perturbations produced during inflation are known to be Gaussian with small corrections. This allows the late time wave function to be written as a power series expansion of the form

$$
\begin{aligned}
\psi[\chi(\boldsymbol{x})]= & \exp \left(-\frac{1}{2} \int d^{3} x d^{3} y \chi(\boldsymbol{x}) \chi(\boldsymbol{y})\langle\hat{O}(\boldsymbol{x}) \hat{O}(\boldsymbol{y})\rangle\right. \\
& \left.+\frac{1}{6} \int d^{3} x d^{3} y d^{3} z \chi(\boldsymbol{x}) \chi(\boldsymbol{y}) \chi(\boldsymbol{z})\langle\hat{O}(\boldsymbol{x}) \hat{O}(\boldsymbol{y}) \hat{O}(\boldsymbol{z})\rangle+\cdots\right) .
\end{aligned}
$$

Here $\chi$ stands for a generic perturbation which could be a scalar or tensor perturbation. The ellipses stand for higher order terms involving more powers of $\phi$. The coefficients $\langle\hat{O}(\boldsymbol{x}) \hat{O}(\boldsymbol{y})\rangle,\langle\hat{O}(\boldsymbol{x}) \hat{O}(\boldsymbol{y}) \hat{O}(\boldsymbol{z})\rangle$ etc. are for now just functions which determine the correlators.

The expression above is schematic. In the case at hand there are two kinds of perturbations, scalar and tensor. Working in the gauge described in subsection 2.1.2 these are $\delta \phi, \gamma_{i j}$. With a suitable choice of normalization the wave function will then take the form

$$
\begin{aligned}
\psi\left[\delta \phi, \gamma_{i j}\right] & =\exp \left[\frac { M _ { p l } ^ { 2 } } { H ^ { 2 } } \left(-\frac{1}{2} \int d^{3} x d^{3} y \delta \phi(\boldsymbol{x}) \delta \phi(\boldsymbol{y})\langle O(\boldsymbol{x}) O(\boldsymbol{y})\rangle\right.\right. \\
& -\frac{1}{2} \int d^{3} x d^{3} y \gamma_{i j}(\boldsymbol{x}) \gamma_{k l}(\boldsymbol{y})\left\langle T^{i j}(\boldsymbol{x}) T^{k l}(\boldsymbol{y})\right\rangle \\
& \left.\left.-\frac{1}{4} \int d^{3} x d^{3} y d^{3} z \delta \phi(\boldsymbol{x}) \delta \phi(\boldsymbol{y}) \gamma_{i j}(\boldsymbol{z})\left\langle O(\boldsymbol{x}) O(\boldsymbol{y}) T^{i j}(\boldsymbol{z})\right\rangle+\cdots\right)\right] .
\end{aligned}
$$

The ellipses stand for additional terms of various kinds involving three powers of the perturbations with appropriate coefficient functions and then higher order terms.

Note, in our notation every additional power of the scalar perturbation is accompanied by an additional factor of $O(\boldsymbol{x})$ in the coefficient functions and every additional power of the tensor perturbation is accompanied by an additional factor of $T_{i j}(\boldsymbol{x})$. We will soon see that the coefficient functions transform under the symmetries in the same way as correlation functions involving a scalar operator and the stress energy tensor in a 3 dimensional Euclidean conformal field theory.

In this paper our interest will be on the last term in the RHS of (2.24). Together with the two point functions, this term determines the three point correlator of interest to us.

\subsection{Symmetries and Their Consequences}

We have seen that the wave function at late times is a functional of the late time values of the perturbations. Schematically we can write

$$
\psi[\chi(\boldsymbol{x})]=\int^{\chi(\boldsymbol{x})} D \chi e^{i S},
$$

where $\chi$ again stands for the value a generic perturbation takes at late time and the action for any configuration is denoted by $S$. We would now like to derive constraints imposed by symmetries on this wavefunction. 
Before doing so it is worth considering the boundary conditions in the path integral in more detail. We will consider inflation with the standard Bunch-Davies boundary conditions in the far past, when the modes of interest had a wavelength much shorter than the Hubble scale. At these early times the short wavelengths of the modes makes them insensitive to the geometry of de Sitter space and they essentially propagate as if in Minkowski spacetime. The Bunch Davies vacuum corresponds to taking the modes to be in the Minkowski vacuum at early enough time.

An elegant way to impose this boundary condition in the path integral above, as discussed in [11], is as follows. Consider de Sitter space in conformal coordinates,

$$
d s^{2}=\frac{1}{\eta^{2}}\left(-d \eta^{2}+\left(d x_{i}\right)^{2}\right)
$$

with the far past being $\eta \rightarrow-\infty$, and late time being $\eta \rightarrow 0$. Continue $\eta$ so that it acquires a small imaginary part $\eta \rightarrow \eta(1-i \epsilon), \epsilon>0$. Then the Bunch Davies boundary condition is correctly imposed if the path integral is done over configurations which vanish at early times when $\eta \rightarrow-\infty(1-i \epsilon)$. Note that in general the resulting path integral is over complex field configurations.

As an example, consider a free field $\phi$ satisfying the equation

$$
\nabla^{2} \phi=0
$$

A mode with momentum $\boldsymbol{k}$ is of the form, $\phi=f_{\boldsymbol{k}}(\eta) e^{i \boldsymbol{k} \cdot \boldsymbol{x}}$, where

$$
f_{\boldsymbol{k}}=c_{1}(1-i k \eta) e^{i k \eta}+c_{2}(1+i k \eta) e^{-i k \eta}
$$

and $k \equiv|\vec{k}|$. Requiring that the solution vanish when $\eta \rightarrow-\infty(1-i \epsilon)$, sets $c_{2}=0$ and requiring $f_{\boldsymbol{k}}$ equals the boundary value, $f_{\boldsymbol{k}}=f_{\boldsymbol{k}}^{0}$ at the late time $\eta=\eta_{c}$, gives

$$
f_{\boldsymbol{k}}=f_{\boldsymbol{k}}^{0} \frac{(1-i k \eta) e^{i k \eta}}{\left(1-i k \eta_{c}\right) e^{i k \eta_{c}}}
$$

Since $f_{\boldsymbol{k}} \neq f_{-\boldsymbol{k}}^{*}$ the resulting field configuration is complex.

We are now ready to return to our discussion of the constraints imposed by symmetries on the wave function. What is important for this purpose, as far as the boundary conditions in the far past are concerned, is that the field configurations we sum over in the path integral vanish in the far past.

Consider in fact a general situation where we have a wave function of the form (2.25) for a general set of fields $\chi$, with some boundary condition in the far past. Now if the system has a symmetry which keeps the action and the measure invariant and which also preserves the boundary conditions in the far past and if under the symmetry the boundary value of the field $\chi$ transforms as follows

$$
\chi(\boldsymbol{x}) \rightarrow \chi^{\prime}(\boldsymbol{x}),
$$


then it follows from the definition of the wave function (2.25) that $\psi[\chi]$ satisfies the condition

$$
\psi[\chi(\boldsymbol{x})]=\psi\left[\chi^{\prime}(\boldsymbol{x})\right]
$$

and is invariant under the symmetry.

For the case at hand where we work with de Sitter space, the symmetry group is the conformal group $S O(1,4)$ of isometries discussed above. Being isometries, the action and measure are invariant under it on account of reparameterization invariance. The boundary condition in the far past corresponding to the Bunch Davies vacuum is that the fields vanish. This is indeed preserved by the conformal transformations since the field transform homogeneously under these symmetries. For tensor perturbations this is all we need to use the general argument above. It follows that the wave function must be invariant under a change of the boundary values of the tensor perturbations which arise due to conformal transformations. As we will see shortly this implies that the coefficient functions, which we have suggestively denoted as $\left\langle T_{i j} T_{k l}\right\rangle$ etc., behave exactly like the correlations functions of the stress energy tensor of a three dimensional conformal field theory under conformal transformations. It is true, as we discussed above, that conformal invariance is broken slightly during inflation but this leads to only subleading corrections in the tensor mode correlations.

For the scalar mode the situation is a little more complicated. In pure de Sitter space, without the inflaton, the scalar perturbation in the metric $\zeta,(2.16)$, is pure gauge. In the presence of the inflaton there is a genuine scalar perturbation. However as (2.21), (2.22) which relates the perturbations in the two gauges discussed in section 2.1 shows, the slow roll parameter $\epsilon$ which is non-zero due to the breaking of conformal invariance is then involved in the definition of the scalar perturbation itself. This can make it confusing to apply the consequences of the small breaking of conformal invariance to the scalar sector.

The simplest way to proceed is to work in the second gauge discussed in subsection 2.1.2, where $\zeta=0$. The scalar perturbation is then just the fluctuation in the scalar field. To leading order in the slow-roll parameters these fluctuations can be calculated in de Sitter space and the time evolution of the inflaton can be neglected for this process. As a result the full set of perturbations, scalar and tensor, with Bunch-Davies boundary conditions, then meet the conditions of the general argument given above and we learn that the wave function must be invariant under conformal transformations of the boundary values of these perturbations.

Once the results are obtained in this gauge one can always transform to other gauges, in particular the first gauge considered in subsection 2.1.1 where $\zeta$ is non-vanishing. In fact this is very convenient to do for purposes of following the evolution of the scalar mode after the end of inflation. Since $\zeta$ is related to $\delta \phi$ by $(2.21)$ the resulting correlation functions will depend on the breaking of conformal invariance even to leading order but this dependence arises solely due to the relation (2.21) and is easily obtained.

Before proceeding let us note that the discussion above has a direct parallel with what happens in a conformal field theory which is deformed by adding a perturbation

$$
\delta S=\int g O
$$


which breaks conformal symmetry slightly. Due to this breaking the trace of the stress tensor $T_{i}^{i}$ does not vanish anymore and instead satisfies the relation

$$
T_{i}^{i}=\beta(g) O
$$

where $\beta(g)$ is the beta function for the coupling in (2.32). To leading order in the breaking correlation functions for $T_{i}^{i}$ can be obtained by first calculating those of $O$ in the CFT (without any breaking) and then transforming these to correlation functions for $T_{i}^{i}$ using (2.33).

\subsection{Constraints on Coefficient Functions}

Let us now work out the constraints imposed by conformal symmetries on the coefficient functions which arise in the expansion of the wave function (2.23) in more detail. It is easy to see that the constraints of translational invariance make the coefficient functions also translationally invariant. Under rotations in the $x^{i}$ directions the wave function will be invariant if $O(\boldsymbol{x})$ transforms like a scalar and $T_{i j}$ like a two-index tensor within coefficient functions.

Next we come to the scale transformation and special conformal transformations. Under the scale transformation (2.12) the scalar perturbation transforms by

$$
\delta \phi(\boldsymbol{x}, t) \rightarrow \delta \phi^{\prime}(\boldsymbol{x}, t)=\delta \phi\left(\frac{\boldsymbol{x}}{\lambda}, t+\frac{1}{H} \log (\lambda)\right)
$$

At late times $\delta \phi$ becomes independent of $t$, as a result this equation becomes

$$
\delta \phi(\boldsymbol{x}) \rightarrow \delta \phi^{\prime}(\boldsymbol{x})=\delta \phi\left(\frac{\boldsymbol{x}}{\lambda}\right) .
$$

In particular this is true for the boundary value of $\delta \phi$ as well.

As a result, suppressing the dependence on tensor modes for the moment, we learn that the wavefunction must satisfy the conditions

$$
\psi[\delta \phi(\boldsymbol{x})]=\psi\left[\delta \phi^{\prime}(\boldsymbol{x})\right]=\psi\left[\delta \phi\left(\frac{\boldsymbol{x}}{\lambda}\right)\right] .
$$

As mentioned above every additional factor of $\delta \phi(\boldsymbol{x})$ in the expansion of the wave function involves an additional factor of $O(\boldsymbol{x})$ in the corresponding coefficient function and also an integral over the spatial position of $\delta \phi(\boldsymbol{x})$. Thus schematically speaking the wave function will satisfy the condition $(2.36)$ if

$$
\int d^{3} x \delta \phi^{\prime}(\boldsymbol{x}) O(\boldsymbol{x})=\int d^{3} x \delta \phi(\boldsymbol{x}) O(\boldsymbol{x}),
$$

where more correctly we mean the coefficient functions involving $O(\boldsymbol{x})$, rather that $O(\boldsymbol{x})$ itself. This leads to the condition

$$
\int d^{3} x \lambda^{3} \delta \phi(\boldsymbol{x}) O(\lambda \boldsymbol{x})=\int d^{3} x \delta \phi(\boldsymbol{x}) O(\boldsymbol{x}) .
$$


(In deriving this relation we first change variables in the middle expression of (2.37) to $\boldsymbol{y}=\frac{\boldsymbol{x}}{\lambda}$ and then change $\boldsymbol{y}$ to $\boldsymbol{x}$ since it is a dummy variable of integration.) Since (2.38) is true for an arbitrary function $\delta \phi(\boldsymbol{x})$ we learn that coefficient functions are invariant under the replacement

$$
O(\boldsymbol{x}) \rightarrow \lambda^{3} O(\lambda \boldsymbol{x}) .
$$

Or in infinitesimal form if $\lambda=1+\epsilon$,

$$
O(\boldsymbol{x}) \rightarrow O(\boldsymbol{x})+\epsilon \delta O(\boldsymbol{x}),
$$

with

$$
\delta O(\boldsymbol{x})=3 O(\boldsymbol{x})+x^{i} \partial_{i} O(\boldsymbol{x}) .
$$

This is exactly the condition that would arise due to scale invariance if the coefficient functions were the correlation functions in a conformal field theory with $O(\boldsymbol{x})$ being an operator of dimension 3 . Note that in 3 dimensions this makes $O(\boldsymbol{x})$ marginal.

A similar argument for the tensor perturbation shows that under the scaling transformation, (2.12), the boundary value of the tensor perturbation transforms like ${ }^{7}$

$$
\gamma_{i j}(\boldsymbol{x}) \rightarrow \gamma_{i j}^{\prime}(\boldsymbol{x})=\gamma_{i j}\left(\frac{\boldsymbol{x}}{\lambda}\right) .
$$

This is entirely analogous to (2.35) and a similar argument leads to the conclusion that $T_{i j}$ must behave like an operator of dimension 3 under scaling transformations for the wave function to be invariant under it.

Finally we consider special conformal transformations. At late times when $e^{-H t} \rightarrow 0$ we see from (2.13) that the $x^{i}$ coordinates transform as

$$
\begin{aligned}
& x^{i} \rightarrow x^{i}+\delta x^{i}, \\
& \delta x^{i}=x^{2} b^{i}-2 x^{i}(\boldsymbol{x} \cdot \boldsymbol{b}) .
\end{aligned}
$$

Henceforth we will use notation where $(\boldsymbol{a} \cdot \boldsymbol{b}) \equiv a^{i} b_{i}$ and also raise and lower indices along the spatial directions using the flat metric $\delta_{i j}$.

The boundary value of the scalar field perturbation transforms under this as

$$
\delta \phi(\boldsymbol{x}) \rightarrow \delta \phi^{\prime}(\boldsymbol{x})=\delta \phi\left(x^{i}-\delta x^{i}\right) .
$$

Arguing as in the case of the scale transformation above we then learn that for the wave function to be invariant coefficient functions must be invariant when

$$
\begin{aligned}
O(\boldsymbol{x}) & \rightarrow O(\boldsymbol{x})+\delta O(\boldsymbol{x}), \\
\delta O(\boldsymbol{x}) & =-6(\boldsymbol{x} \cdot \boldsymbol{b}) O(\boldsymbol{x})+D O(\boldsymbol{x}), \\
D & =x^{2}(\boldsymbol{b} \cdot \boldsymbol{\partial})-2(\boldsymbol{b} \cdot \boldsymbol{x})(\boldsymbol{x} \cdot \boldsymbol{\partial}) .
\end{aligned}
$$

\footnotetext{
${ }^{7}$ The reader might find this puzzling at first since the metric should transform as a tensor under the coordinate transformation (2.12). In fact the metric $h_{i j},(2.16)$, does transform like a tensor and goes to $h_{i j}(\boldsymbol{x}) \rightarrow \frac{1}{\lambda^{2}} h_{i j}\left(\frac{\boldsymbol{x}}{\lambda}\right)$. However $\gamma_{i j}$ is related to $h_{i j}$ after multiplying by an additional factor of $a^{2},(2.17)$. Since $t$ shifts, (2.12), the $a^{2}$ factor also changes resulting in the transformation rule (2.42).
} 
This is exactly the transformation of an operator of dimension 3 under special conformal transformations. Similarly from the transformation of the tensor mode we learn that the coefficient functions must be invariant when

$$
\begin{aligned}
T_{i j}(\boldsymbol{x}) & \rightarrow T_{i j}+\delta T_{i j}, \\
\delta T_{i j} & =-6(\boldsymbol{x} \cdot \boldsymbol{b}) T_{i j}+2 \hat{M}_{i}^{k} T_{k j}+2 \hat{M}_{j}^{k} T_{i k}-D T_{i j}, \\
\hat{M}_{i}^{k} & \equiv 2\left(x^{k} b^{i}-x^{i} b^{k}\right) .
\end{aligned}
$$

These agree with the transformation rules for the stress energy tensor of a $3 \mathrm{~d}$ CFT and also agree with eq.(4.9) in [13].

The stress energy tensor of a CFT also satisfies one additional condition - it is conserved. This gives rise to Ward identities that must be satisfied by correlations functions in the CFT involving the stress energy tensor. The same conditions also arise for the coefficient functions at hand here. The wave function must be reparameterization invariant with respect to general coordinate transformations,

$$
x^{i} \rightarrow x^{i}+v^{i}
$$

under which the metric and scalar perturbations transform as

$$
\begin{aligned}
\gamma_{i j} & \rightarrow \gamma_{i j}-\nabla_{i} v_{j}-\nabla_{j} v_{i} \\
\delta \phi & \rightarrow \delta \phi-v^{k} \partial_{k} \delta \phi .
\end{aligned}
$$

Invariance of the wave function $\psi\left[\gamma_{i j}, \delta \phi\right]$ then leads to the condition

$$
\int d^{3} x v^{j} \partial_{x^{i}}\left\langle T_{i j}(\boldsymbol{x}) \hat{O}\left(\boldsymbol{y}_{\mathbf{1}}\right) \hat{O}\left(\boldsymbol{y}_{\mathbf{2}}\right) \cdots \hat{O}\left(\boldsymbol{y}_{\boldsymbol{n}}\right)\right\rangle=-\sum_{i}\left\langle\hat{O}\left(\boldsymbol{y}_{\mathbf{1}}\right) \cdots \delta \hat{O}\left(\boldsymbol{y}_{\boldsymbol{i}}\right) \cdots \hat{O}\left(\boldsymbol{y}_{\boldsymbol{n}}\right)\right\rangle
$$

where $\hat{O}$ is a schematic notation standing for both $T_{i j}, O$, and $\delta \hat{O}\left(\boldsymbol{y}_{\boldsymbol{i}}\right)$ is the change in operator $\hat{O}\left(\boldsymbol{y}_{\boldsymbol{i}}\right)$ at the point $\boldsymbol{y}_{\boldsymbol{i}}$. In particular when $\hat{O}=O$ is a scalar we get for the three point function

$$
\begin{aligned}
\partial_{x^{i}}\left\langle T_{i j}(\boldsymbol{x}) O\left(\boldsymbol{y}_{\mathbf{1}}\right) O\left(\boldsymbol{y}_{\mathbf{2}}\right)\right\rangle= & {\left[\partial_{x^{j}} \delta^{3}\left(\boldsymbol{x}-\boldsymbol{y}_{\mathbf{1}}\right)\right]\left\langle O\left(\boldsymbol{y}_{\mathbf{1}}\right) O\left(\boldsymbol{y}_{\mathbf{2}}\right)\right\rangle } \\
& +\left[\partial_{x^{j}} \delta^{3}\left(\boldsymbol{x}-\boldsymbol{y}_{\mathbf{2}}\right)\right]\left\langle O\left(\boldsymbol{y}_{\mathbf{1}}\right) O\left(\boldsymbol{y}_{\mathbf{2}}\right)\right\rangle .
\end{aligned}
$$

To summarize, the coefficient functions which arise in the wave function (2.23) satisfy all the symmetry properties of correlations functions involving a scalar operator of dimension 3 and the stress energy tensor in a conformal field theory. Namely, they are invariant under the conformal symmetry group $S O(1,4)$ and satisfy the Ward identities due to conservation of the stress energy tensor.

Let us end this section by noting that readers familiar with the AdS/CFT correspondence will hardly find the connection discussed above between the coefficient functions and the correlation functions of a CFT surprising. For the wave function in the inflationary context (2.25) is the analogue of the bulk partition function in the AdS/CFT correspondence which in turn equals the CFT partition function in the presence of sources. 


\section{Constraints of Conformal Invariance on the Correlation Function}

In this section we will discuss how the correlation function (1.1) is constrained by the symmetries. This correlation function is obtained from the coefficient function, $\left\langle O O T_{i j}\right\rangle$, of the wave function in (2.23). We have argued in the previous section that as far as symmetries are concerned the coefficient functions behave in exactly the same manner as corresponding correlation functions of a CFT. In our discussion below we will find it convenient to adopt the language of CFT. We remind the reader that this is only a kind of short-hand for analyzing the consequences of symmetries. In particular, we will not be assuming any kind of deeper $\mathrm{dS} / \mathrm{CFT}$ type relation in our analysis.

We will work in momentum space below. We derive our constraints in two ways. The first is to directly act with the generators of conformal transformations on the momentum space correlator. The other is to translate the correlator into the spinor-helicity formalism, and then use the conformal generators in terms of those variables. Of course, we obtain the same differential equations with both approach. The spinor-helicity formalism has the disadvantage of being a little more technical but leads to the result a little more directly. The reader who is unfamiliar with the spinor-helicity formalism can skip $\S 3.2$ and $\S 3.3$ on a first reading and proceed from $\S 3.1$ directly to $\S 4$.

\section{Notation:}

Before proceeding let us list our conventions. We denote the three momentum by $\boldsymbol{k}$ below. Its magnitude will be denoted simply by $k \equiv|\boldsymbol{k}|$. Components will be denoted by $k_{i}, i=1, \ldots, 3$ and indices will be raised and lowered by the flat space metric $\delta_{i j}$.

\subsection{Direct Momentum Space Analysis}

In our conventions the momentum space scalar operator is given by

$$
O(\boldsymbol{k}) \equiv \int d^{3} x O(\boldsymbol{x}) e^{-i \boldsymbol{k} \cdot \boldsymbol{x}}
$$

and similarly for $T_{i j}(\boldsymbol{k})$.

Translational and rotational invariance allows us to express the correlators in the form

$$
\begin{aligned}
\left\langle O\left(\boldsymbol{k}_{1}\right) O\left(\boldsymbol{k}_{2}\right) T_{i j}\left(\boldsymbol{k}_{3}\right)\right\rangle & =\left[k_{1 i} k_{1 j} f_{1}\left(k_{1}, k_{2}, k_{3}\right)+k_{2 i} k_{2 j} f_{1}\left(k_{2}, k_{1}, k_{3}\right)\right. \\
& +\left(k_{1 i} k_{2 j}+k_{2 i} k_{1 j}\right) f_{2}\left(k_{1}, k_{2}, k_{3}\right) \\
& \left.+\delta_{i j} f_{3}\left(k_{1}, k_{2}, k_{3}\right)\right](2 \pi)^{3} \delta^{3}\left(\sum_{i} \boldsymbol{k}_{i}\right) .
\end{aligned}
$$

The overall delta function arises due to translational invariance. In the discussion below we will use $M_{i j}\left(\boldsymbol{k}_{1}, \boldsymbol{k}_{2}, \boldsymbol{k}_{3}\right)$ to denote the correlation function without the overall delta function factor,

$$
\left\langle O\left(\boldsymbol{k}_{1}\right) O\left(\boldsymbol{k}_{2}\right) T_{i j}\left(\boldsymbol{k}_{3}\right)\right\rangle=M_{i j}\left(\boldsymbol{k}_{1}, \boldsymbol{k}_{2}, \boldsymbol{k}_{3}\right)(2 \pi)^{3} \delta\left(\sum_{i} \boldsymbol{k}_{i}\right)
$$


The three functions $f_{1}, f_{2}, f_{3}$ in (3.2) at first sight could have also depended on inner products $\boldsymbol{k}_{\mathbf{1}} \cdot \boldsymbol{k}_{\mathbf{2}}$ etc. However using momentum conservation these can be expressed in terms of the three scalars $k_{i}$. For example

$$
\boldsymbol{k}_{1} \cdot \boldsymbol{k}_{\mathbf{2}}=\frac{1}{2}\left(k_{3}^{2}-k_{1}^{2}-k_{2}^{2}\right) .
$$

The correlator is symmetric under the exchange of $\boldsymbol{k}_{1} \leftrightarrow \boldsymbol{k}_{2}$. As a result $f_{2}, f_{3}$ are symmetric under the exchange of their first two arguments. Since the operators $O$ and $T_{i j}$ are dimension 3 in position space and thus dimension 0 in momentum space, scale invariance tells us that the $f_{i}$ 's are dimension 1 .

Next we come to the non-trivial constraints due to special conformal transformations. The transformation in position space of the operators $O$ and $T_{i j}$ under an infinitesimal special conformal transformation with parameter $b_{i}$ is given in (2.47) and (2.50) respectively. In momentum space these take the form,

$$
\begin{aligned}
\delta O(\boldsymbol{k}) & =-\tilde{D} O(\boldsymbol{k}), \\
\delta T_{i j}(\boldsymbol{k}) & =2 \tilde{M}_{i}^{l} T_{l j}+2 \tilde{M}_{j}^{l} T_{i l}-\tilde{D} T_{i j}, \\
\tilde{M}_{i}^{l} & \equiv b^{l} \partial_{k^{i}}-b^{i} \partial_{k^{l}}, \\
\tilde{D} & \equiv(\boldsymbol{b} \cdot \boldsymbol{k}) \partial_{k^{i}} \partial_{k^{i}}-2 k_{j} \partial_{k_{j}}\left(\boldsymbol{b} \cdot \boldsymbol{\partial}_{\boldsymbol{k}}\right) .
\end{aligned}
$$

These expressions agree with eq.(4.12) in [13] and in fact we have chosen essentially the same conventions to try and ensure readability.

The condition for invariance of the correlator is

$$
\left\langle\delta O\left(\boldsymbol{k}_{1}\right) O\left(\boldsymbol{k}_{2}\right) T_{i j}\left(\boldsymbol{k}_{3}\right)\right\rangle+\left\langle O\left(\boldsymbol{k}_{1}\right) \delta O\left(\boldsymbol{k}_{2}\right) T_{i j}\left(\boldsymbol{k}_{3}\right)\right\rangle+\left\langle O\left(\boldsymbol{k}_{1}\right) O\left(\boldsymbol{k}_{2}\right) \delta T_{i j}\left(\boldsymbol{k}_{3}\right)\right\rangle=0 .
$$

As was argued in [13] all terms involving derivatives that act on the overall momentum conserving delta function sum to zero so we will henceforth neglect the effect of the derivative operators acting on the delta function.

Defining the operator

$$
\Theta(k) \equiv-\frac{2}{k} \frac{\partial}{\partial k}+\frac{\partial^{2}}{\partial k^{2}}
$$

where $k \equiv|\boldsymbol{k}|$ one can then show after some algebra that

$$
\begin{aligned}
& \left\langle\delta O\left(\boldsymbol{k}_{1}\right) O\left(\boldsymbol{k}_{2}\right) T_{i j}\left(\boldsymbol{k}_{3}\right)\right\rangle=-2\left(\boldsymbol{b} \cdot \boldsymbol{k}_{\mathbf{1}}\right) \delta_{i j} f_{1}+2\left(b_{i} k_{1 j}+b_{j} k_{1 i}\right)\left(1+k_{1} \partial_{k_{1}}\right) f_{1} \\
& +2\left(b_{i} k_{2 j}+b_{j} k_{2 i}\right) k_{1} \partial_{k_{1}} f_{2}+\left(\boldsymbol{b} \cdot \boldsymbol{k}_{\mathbf{1}}\right) \Theta\left(k_{1}\right)\left[f_{1} k_{1 i} k_{1 j}+f_{1}^{T} k_{2 i} k_{2 j}+f_{2}\left(k_{1 i} k_{2 j}+k_{2 i} k_{1 j}\right)+f_{3} \delta_{i j}\right] .
\end{aligned}
$$

Here we have omitted the overall delta function. We have also introduced the notation

$$
f_{1}^{T}\left(k_{1}, k_{2}, k_{3}\right) \equiv f_{1}\left(k_{2}, k_{1}, k_{3}\right) .
$$


At this stage it is useful to contract the LHS of (3.11) with the symmetric (real) polarization tensor $e_{i j}^{s}$ which is traceless and transverse to $\boldsymbol{k}_{3}$,

$$
e_{i}^{s, i}=e_{i j}^{s} k_{3}^{i}=0
$$

The $s$ here indicates that there are two possible choices for this tensor. This gives

$$
\begin{aligned}
\left\langle\delta O\left(\boldsymbol{k}_{1}\right) O\left(\boldsymbol{k}_{2}\right) T_{i j}\left(\boldsymbol{k}_{3}\right)\right\rangle e^{s, i j}= & 4 b_{i} k_{1 j} e^{s, i j}\left[\left(1+k_{1} \partial_{k_{1}}\right) f_{1}-k_{1} \partial_{k_{1}} f_{2}\right] \\
& +\left(\boldsymbol{b} \cdot \boldsymbol{k}_{\mathbf{1}}\right) \Theta\left(k_{1}\right)\left(2 f_{2}-f_{1}-f_{1}^{T}\right) k_{1 i} k_{2 j} e^{s, i j}
\end{aligned}
$$

where we have used the condition

$$
e^{s, i j} k_{1 i}=-e^{s, i j} k_{2 i}=0
$$

Similarly we get

$$
\begin{aligned}
\left\langle O\left(\boldsymbol{k}_{1}\right) \delta O\left(\boldsymbol{k}_{2}\right) T_{i j}\left(\boldsymbol{k}_{3}\right)\right\rangle e^{s, i j}= & -4 b_{i} k_{1 j} e^{s, i j}\left[\left(1+k_{2} \partial_{k_{2}}\right) f_{1}^{T}-k_{2} \partial_{k_{2}} f_{2}\right] \\
& +\left(\boldsymbol{b} \cdot \boldsymbol{k}_{\mathbf{2}}\right) \Theta\left(k_{2}\right)\left(2 f_{2}-f_{1}-f_{1}^{T}\right) k_{1 i} k_{2 j} e^{s, i j}
\end{aligned}
$$

And also

$$
\begin{aligned}
\left\langle O\left(\boldsymbol{k}_{1}\right) O\left(\boldsymbol{k}_{2}\right) \delta T_{i j}\left(\boldsymbol{k}_{3}\right)\right\rangle e^{s, i j}= & -\frac{4}{k_{3}} b_{i} k_{1 j} e^{s, i j}\left[\left(\boldsymbol{k}_{\mathbf{3}} \cdot \boldsymbol{k}_{\mathbf{1}}\right) \partial_{k_{3}}\left(f_{1}-f_{2}\right)-\left(\boldsymbol{k}_{\mathbf{3}} \cdot \boldsymbol{k}_{\mathbf{2}}\right) \partial_{k_{3}}\left(f_{1}^{T}-f_{2}\right)\right] \\
& +\boldsymbol{b} \cdot \boldsymbol{k}_{\mathbf{3}} \Theta\left(k_{3}\right)\left(2 f_{2}-f_{1}-f_{1}^{T}\right)
\end{aligned}
$$

Adding (3.14), (3.16) and (3.17) and setting the total change to vanish finally gives the equation

$$
\begin{aligned}
& 4 b_{i} k_{1 j} e^{s, i j}[\left(1+k_{1} \partial_{k_{1}}\right) f_{1}-\left(1+k_{2} \partial_{k_{2}}\right) f_{1}^{T}+\left(k_{2} \partial_{k_{2}}-k_{1} \partial_{k_{1}}\right) f_{2} \\
&\left.-\frac{\left(\boldsymbol{k}_{\mathbf{3}} \cdot \boldsymbol{k}_{\mathbf{1}}\right)}{k_{3}} \partial_{k_{3}}\left(f_{1}-f_{2}\right)+\frac{\left(\boldsymbol{k}_{\mathbf{3}} \cdot \boldsymbol{k}_{\mathbf{2}}\right)}{k_{3}} \partial_{k_{3}}\left(f_{1}^{T}-f_{2}\right)\right] \\
&+k_{1 i} k_{2 j} e^{s, i j}\left[\left(\boldsymbol{b} \cdot \boldsymbol{k}_{\mathbf{1}}\right) \Theta\left(k_{1}\right)+\left(\boldsymbol{b} \cdot \boldsymbol{k}_{\mathbf{2}}\right) \Theta\left(k_{2}\right)+\left(\boldsymbol{b} \cdot \boldsymbol{k}_{\mathbf{3}}\right) \Theta\left(k_{3}\right)\right]\left(2 f_{2}-f_{1}-f_{1}^{T}\right)=0 .
\end{aligned}
$$

This is the main equation we will use to derive the constraints imposed by the special conformal transformations.

There are three linearly independent values that $\boldsymbol{b}$ can take in (3.18). Choosing $\boldsymbol{b} \propto \boldsymbol{k}_{3}$ gives

$$
\left[\boldsymbol{k}_{3} \cdot \boldsymbol{k}_{1} \Theta\left(k_{1}\right)+\left(\boldsymbol{k}_{\mathbf{3}} \cdot \boldsymbol{k}_{\mathbf{2}}\right) \Theta\left(k_{2}\right)+k_{3}^{2} \Theta\left(k_{3}\right)\right] S\left(k_{1}, k_{2}, k_{3}\right)=0,
$$

where

$$
S\left(k_{1}, k_{2}, k_{3}\right)=\frac{1}{2}\left[f_{1}\left(k_{1}, k_{2}, k_{3}\right)+f_{1}\left(k_{2}, k_{1}, k_{3}\right)-2 f_{2}\left(k_{1}, k_{2}, k_{3}\right)\right] .
$$


Choosing $\boldsymbol{b} \propto \boldsymbol{k}_{1 \perp}=\boldsymbol{k}_{1}-\boldsymbol{k}_{3} \frac{\left(\boldsymbol{k}_{1} \cdot \boldsymbol{k}_{3}\right)}{k_{3}^{2}}$ gives

$$
\begin{aligned}
& 4\left[\frac{-\boldsymbol{k}_{\mathbf{2}} \cdot \boldsymbol{k}_{\mathbf{3}}}{k_{3}^{2}} k_{1} \partial_{k_{1}} S+\frac{\boldsymbol{k}_{\mathbf{1}} \cdot \boldsymbol{k}_{\mathbf{3}}}{k_{3}^{2}}\right.\left.k_{2} \partial_{k_{2}} S-\frac{\left(k_{1}^{2}-k_{2}^{2}\right)}{k_{3}^{2}} S+\frac{3}{2} \frac{\left(k_{1}^{3}-k_{2}^{3}\right)}{k_{3}^{2}}\right] \\
&-\left(k_{1}^{2}-\frac{\left(\boldsymbol{k}_{\mathbf{3}} \cdot \boldsymbol{k}_{\mathbf{1}}\right)^{2}}{k_{3}^{2}}\right)\left(\Theta\left(k_{1}\right)-\Theta\left(k_{2}\right)\right) S=0,
\end{aligned}
$$

as shown in Appendix B. The term inhomogeneous in $S$ above arises due to the use of the Ward identity for conservation of the stress tensor. We take the two-point function of the scalar $O(k)$ to be normalized so that

$$
\left\langle O\left(k_{1}\right) O\left(k_{2}\right)\right\rangle=(2 \pi)^{3} \delta\left(\boldsymbol{k}_{1}+\boldsymbol{k}_{2}\right)\left|\boldsymbol{k}_{1}\right|^{3} .
$$

The Ward identity for conservation of the stress tensor, (2.56) then takes the form

$$
M_{i j} k_{3}^{j}=-k_{1}^{3} k_{1}^{j}-k_{2}^{3} k_{2}^{j}
$$

where $M_{i j}$ is defined in (3.3).

Finally we can choose $\boldsymbol{b}$ to be orthogonal to all the $\boldsymbol{k}_{i}$ 's so that $\boldsymbol{b} \cdot \boldsymbol{k}_{i}=0$. For a suitable choice of polarization $b_{i} k_{1 j} e^{s, i j}$ will not vanish and as discussed in Appendix B (3.18) then becomes

$$
-\left(\boldsymbol{k}_{\mathbf{2}} \cdot \boldsymbol{k}_{\mathbf{3}}\right) k_{1} \partial_{k_{1}} S+\left(\boldsymbol{k}_{\mathbf{1}} \cdot \boldsymbol{k}_{\mathbf{3}}\right) k_{2} \partial_{k_{2}} S-\left(k_{1}^{2}-k_{2}^{2}\right) S+\frac{3}{2}\left(k_{1}^{3}-k_{2}^{3}\right)=0
$$

Subtracting (3.21) and (3.24) then gives

$$
\left(\Theta\left(k_{1}\right)-\Theta\left(k_{2}\right)\right) S=0 .
$$

Substituting this in (3.19) then gives

$$
\left(\Theta\left(k_{1}\right)-\Theta\left(k_{3}\right)\right) S=0
$$

Equations (3.24), (3.25) and (3.26) can be taken to be the three final equations which arise because of special conformal invariance.

Before proceeding let us note here that from (3.2), (3.15) and (3.20) we get that

$$
\left\langle O\left(k_{1}\right) O\left(k_{2}\right) T_{i j}\left(k_{3}\right)\right\rangle e^{s, i j}=-2(2 \pi)^{3} \delta\left(\sum_{i} \boldsymbol{k}_{i}\right) e^{s, i j} k_{1 i} k_{2 j} S
$$

where $e_{i j}^{s}$ is a traceless polarization tensor transverse to $\boldsymbol{k}_{3}$.

\subsection{Analysis using the Spinor Helicity Formalism}

We now rederive these differential equations in a second way, using the spinor helicity formalism of [13] and [17]. Our notation is described in detail in Appendix C. 
Although the correlator in (3.3) appears to have several independent components, the use of the Ward identities for the conservation of the stress-tensor and its tracelessness, leave us with only two components. We can extract both of these by considering the quantities:

$$
\begin{aligned}
& M^{+}\left(\boldsymbol{k}_{\mathbf{1}}, \boldsymbol{k}_{\mathbf{2}}, \boldsymbol{k}_{\mathbf{3}}\right)(2 \pi)^{3} \delta\left(\boldsymbol{k}_{\mathbf{1}}+\boldsymbol{k}_{\mathbf{2}}+\boldsymbol{k}_{\mathbf{3}}\right)=\frac{1}{k_{1} k_{2} k_{3}} e_{i j}^{+}\left\langle O\left(\boldsymbol{k}_{\mathbf{1}}\right) O\left(\boldsymbol{k}_{\mathbf{2}}\right) T^{i j}\left(\boldsymbol{k}_{\mathbf{3}}\right)\right\rangle, \\
& M^{-}\left(\left(\boldsymbol{k}_{\mathbf{1}}, \boldsymbol{k}_{\mathbf{2}}, \boldsymbol{k}_{\mathbf{3}}\right)(2 \pi)^{3} \delta\left(\boldsymbol{k}_{\mathbf{1}}+\boldsymbol{k}_{\mathbf{2}}+\boldsymbol{k}_{\mathbf{3}}\right)=\frac{1}{k_{1} k_{2} k_{3}} e_{i j}^{-}\left\langle O\left(\boldsymbol{k}_{\mathbf{1}}\right) O\left(\boldsymbol{k}_{\mathbf{2}}\right) T^{i j}\left(\boldsymbol{k}_{\mathbf{3}}\right)\right\rangle,\right.
\end{aligned}
$$

where $e^{+}$and $e^{-}$are symmetric traceless tensors that are transverse to $\boldsymbol{k}_{\mathbf{3}}$. We caution the reader that these are linear combinations of the real polarization tensors $e^{s}$ that have appeared previously and whenever we use these "circularly polarized" tensors, we put a \pm rather than a $s$ in the superscript. We give explicit expressions for these tensors in Appendix C. The pre-factor of $\frac{1}{k_{1} k_{2} k_{3}}$ is included for convenience.

The momentum space correlators manifestly have an $S O(3)$ symmetry and just this allows us to write

$$
M^{-}\left(\boldsymbol{k}_{\mathbf{1}}, \boldsymbol{k}_{\mathbf{2}}, \boldsymbol{k}_{\mathbf{3}}\right)=\tilde{R}\left(k_{1}, k_{2}, k_{3}\right) \frac{\left\langle\lambda_{3}, \lambda_{1}\right\rangle^{2}\left\langle\lambda_{3}, \lambda_{2}\right\rangle^{2}}{\left\langle\lambda_{1}, \lambda_{2}\right\rangle^{2}} .
$$

We can write a similar expression for $M^{+}$, but this leads to the same constraints, and our analysis can be performed entirely with the expression above. We now need to derive constraints on the function $\tilde{R}$, which depends just on the norms of the momenta.

The constraints of special conformal invariance, in the spinor helicity formalism (see the Appendix for a derivation) can be written as

$$
b_{i} \sigma_{\alpha \dot{\alpha}}^{i} \sum \frac{\partial}{\partial \lambda_{n \alpha}} \frac{\partial}{\partial \bar{\lambda}_{n \dot{\alpha}}} M^{-}=\left(\frac{\left(\boldsymbol{b} \cdot \boldsymbol{k}_{1}\right)}{k_{1}^{2}}+\frac{\left(\boldsymbol{b} \cdot \boldsymbol{k}_{2}\right)}{k_{2}^{2}}\right) M^{-}+W,
$$

where $W$ is the Ward identity term

$$
W=\frac{3 b^{k} e_{k j}^{-} k_{3 i}}{k_{3}^{3}}\left\langle O\left(\boldsymbol{k}_{\mathbf{1}}\right) O\left(\boldsymbol{k}_{\mathbf{2}}\right) T^{i j}\left(\boldsymbol{k}_{\mathbf{3}}\right)\right\rangle+(i \leftrightarrow j) .
$$

Now, notice that

$$
\begin{aligned}
b_{i} \sigma_{\alpha \dot{\alpha}}^{i} \frac{\partial}{\partial \lambda_{1 \alpha}} \frac{\partial}{\partial \bar{\lambda}_{1 \dot{\alpha}}} M^{-}= & b_{i} \sigma_{\alpha \dot{\alpha}}^{i} \frac{\left\langle\lambda_{3}, \lambda_{1}\right\rangle^{2}\left\langle\lambda_{3}, \lambda_{2}\right\rangle^{2}}{\left\langle\lambda_{1}, \lambda_{2}\right\rangle^{2}} \frac{\partial}{\partial \lambda_{1 \alpha}} \frac{\partial}{\partial \bar{\lambda}_{1 \dot{\alpha}}} \tilde{R} \\
& +b_{i} \sigma_{\alpha \dot{\alpha}}^{i}\left(\frac{\partial}{\partial \bar{\lambda}_{1 \dot{\alpha}}} \tilde{R}\right) \frac{\partial}{\partial \lambda_{1 \alpha}} \frac{\left\langle\lambda_{3}, \lambda_{1}\right\rangle^{2}\left\langle\lambda_{3}, \lambda_{2}\right\rangle^{2}}{\left\langle\lambda_{1}, \lambda_{2}\right\rangle^{2}}
\end{aligned}
$$

We see that

$$
\begin{aligned}
\frac{\partial}{\partial \lambda_{1 \alpha}} \frac{\left\langle\lambda_{3}, \lambda_{1}\right\rangle^{2}\left\langle\lambda_{3}, \lambda_{2}\right\rangle^{2}}{\left\langle\lambda_{1}, \lambda_{2}\right\rangle^{2}} & =\left\langle\lambda_{3}, \lambda_{2}\right\rangle^{2}\left(\frac{2\left\langle\lambda_{1}, \lambda_{3}\right\rangle \lambda_{3}^{\alpha}}{\left\langle\lambda_{1}, \lambda_{2}\right\rangle^{2}}-2 \frac{\left\langle\lambda_{1}, \lambda_{3}\right\rangle^{2} \lambda_{2}^{\alpha}}{\left\langle\lambda_{1}, \lambda_{2}\right\rangle^{3}}\right) \\
& =\frac{2\left\langle\lambda_{3}, \lambda_{2}\right\rangle^{3}\left\langle\lambda_{1}, \lambda_{3}\right\rangle}{\left\langle\lambda_{1}, \lambda_{2}\right\rangle^{3}} \lambda_{1}^{\alpha},
\end{aligned}
$$


where we have used the Schouten identity in the last step. Also,

$$
\frac{\partial \tilde{R}}{\partial \bar{\lambda}_{1 \dot{\alpha}}}=\frac{\partial \tilde{R}}{\partial k_{1}} \frac{\partial k_{1}}{\partial \bar{\lambda}_{1 \dot{\alpha}}}=\frac{1}{2} \bar{\sigma}_{0}^{\dot{\alpha} \beta} \lambda_{1 \beta} \frac{\partial \tilde{R}}{\partial k_{1}} .
$$

This tells us that

$$
b_{i} \sigma_{\alpha \dot{\alpha}}^{i}\left(\frac{\partial}{\partial \bar{\lambda}_{1 \dot{\alpha}}} \tilde{R}\right) \frac{\partial}{\partial \lambda_{1 \alpha}} \frac{\left\langle\lambda_{3}, \lambda_{1}\right\rangle^{2}\left\langle\lambda_{3}, \lambda_{2}\right\rangle^{2}}{\left\langle\lambda_{1}, \lambda_{2}\right\rangle^{2}}=2 \frac{\left\langle\lambda_{3}, \lambda_{2}\right\rangle^{3}\left\langle\lambda_{1}, \lambda_{3}\right\rangle}{\left\langle\lambda_{1}, \lambda_{2}\right\rangle^{3}} k_{1}\left(\boldsymbol{b} \cdot \boldsymbol{\epsilon}_{\mathbf{1}}\right) \frac{\partial \tilde{R}}{\partial k_{1}},
$$

where $\epsilon_{1}$ is the transverse and null "polarization vector" defined by (C.11).

Putting this algebra together, we see that

$$
b_{i} \sigma_{\alpha \dot{\alpha}}^{i} \frac{\partial}{\partial \lambda_{1 \alpha}} \frac{\partial}{\partial \bar{\lambda}_{1 \dot{\alpha}}} M^{-}=\frac{\left(\boldsymbol{b} \cdot \boldsymbol{k}_{\mathbf{1}}\right)}{2} \frac{\left\langle\lambda_{3}, \lambda_{1}\right\rangle^{2}\left\langle\lambda_{3}, \lambda_{2}\right\rangle^{2}}{\left\langle\lambda_{1}, \lambda_{2}\right\rangle^{2}} \frac{\partial^{2}}{\partial k_{1}^{2}} \tilde{R}+2 \frac{\left\langle\lambda_{3}, \lambda_{2}\right\rangle^{3}\left\langle\lambda_{1}, \lambda_{3}\right\rangle}{\left\langle\lambda_{1}, \lambda_{2}\right\rangle^{3}} k_{1}\left(\boldsymbol{b} \cdot \boldsymbol{\epsilon}_{\mathbf{1}}\right) \frac{\partial \tilde{R}}{\partial k_{1}} .
$$

By interchanging $1 \leftrightarrow 2$, we see that

$$
b_{i} \sigma_{\alpha \dot{\alpha}}^{i} \frac{\partial}{\partial \lambda_{2 \alpha}} \frac{\partial}{\partial \bar{\lambda}_{2 \dot{\alpha}}} M^{-}=\frac{\left(\boldsymbol{b} \cdot \boldsymbol{k}_{\mathbf{2}}\right)}{2} \frac{\left\langle\lambda_{3}, \lambda_{2}\right\rangle^{2}\left\langle\lambda_{3}, \lambda_{1}\right\rangle^{2}}{\left\langle\lambda_{2}, \lambda_{1}\right\rangle^{2}} \frac{\partial^{2}}{\partial k_{2}^{2}} \tilde{R}+2 \frac{\left\langle\lambda_{3}, \lambda_{1}\right\rangle^{3}\left\langle\lambda_{2}, \lambda_{3}\right\rangle}{\left\langle\lambda_{2}, \lambda_{1}\right\rangle^{3}} k_{2}\left(\boldsymbol{b} \cdot \boldsymbol{\epsilon}_{\mathbf{2}}\right) \frac{\partial \tilde{R}}{\partial k_{2}} .
$$

Turning to the third particle, we note that

$$
\begin{aligned}
\frac{\partial}{\partial \lambda_{3 \alpha}} \frac{\left\langle\lambda_{3}, \lambda_{1}\right\rangle^{2}\left\langle\lambda_{3}, \lambda_{2}\right\rangle^{2}}{\left\langle\lambda_{1}, \lambda_{2}\right\rangle^{2}} & =2 \frac{\left\langle\lambda_{3}, \lambda_{1}\right\rangle\left\langle\lambda_{3}, \lambda_{2}\right\rangle}{\left\langle\lambda_{1}, \lambda_{2}\right\rangle}\left(\lambda_{1}^{\alpha} \frac{\left\langle\lambda_{3}, \lambda_{2}\right\rangle}{\left\langle\lambda_{1}, \lambda_{2}\right\rangle}+\lambda_{2}^{\alpha} \frac{\left\langle\lambda_{3}, \lambda_{1}\right\rangle}{\left\langle\lambda_{1}, \lambda_{2}\right\rangle}\right) \\
& \equiv 2 \frac{\left\langle\lambda_{3}, \lambda_{1}\right\rangle\left\langle\lambda_{3}, \lambda_{2}\right\rangle}{\left\langle\lambda_{1}, \lambda_{2}\right\rangle} \lambda_{4}^{\alpha},
\end{aligned}
$$

where we have defined a new spinor $\lambda_{4}$ in the last step for convenience. This leads to

$$
b_{i} \sigma_{\alpha \dot{\alpha}}^{i} \frac{\partial}{\partial \lambda_{3 \alpha}} \frac{\partial}{\partial \bar{\lambda}_{3 \dot{\alpha}}} M^{-}=\frac{\left(\boldsymbol{b} \cdot \boldsymbol{k}_{3}\right)}{2} \frac{\left\langle\lambda_{3}, \lambda_{2}\right\rangle^{2}\left\langle\lambda_{3}, \lambda_{1}\right\rangle^{2}}{\left\langle\lambda_{2}, \lambda_{1}\right\rangle^{2}} \frac{\partial^{2}}{\partial k_{3}^{2}} \tilde{R}+2 \frac{\left\langle\lambda_{3}, \lambda_{1}\right\rangle\left\langle\lambda_{3}, \lambda_{2}\right\rangle}{\left\langle\lambda_{1}, \lambda_{2}\right\rangle}\left(b_{\alpha \dot{\alpha}} \hat{\lambda}_{3}^{\dot{\alpha}} \lambda_{4}^{\alpha}\right) \frac{\partial \tilde{R}}{\partial k_{3}},
$$

where recall that $b_{\alpha \dot{\alpha}}=b_{i} \sigma_{\alpha \dot{\alpha}}^{i}$, where the sum on $i$ runs only over $0,1,2$.

\subsubsection{Equations for Conformal Invariance}

The choice of $\boldsymbol{b}$ allows us to project this in various directions. It is most convenient to take $\boldsymbol{b}=\boldsymbol{\epsilon}_{\boldsymbol{n}}$, with $n=1,2,3$ in turn.

$\boldsymbol{b} \propto \boldsymbol{\epsilon}_{3}$ : Let us start with $b_{\alpha \dot{\alpha}}=2 \lambda_{3 \alpha} \hat{\lambda}_{3 \dot{\alpha}}=2 k_{3} \epsilon_{3}$. The advantage of this particular case is that the Ward identity term does not contribute for this choice of $\boldsymbol{b}$ and moreover, (3.39) drops out since $\boldsymbol{\epsilon}_{\mathbf{3}} \cdot \boldsymbol{k}_{\mathbf{3}}=\boldsymbol{\epsilon}_{\mathbf{3}} \cdot \boldsymbol{\epsilon}_{\mathbf{4}}=0$. With this choice of $\boldsymbol{b}$, we see that

$$
\begin{aligned}
& k_{1} \boldsymbol{b} \cdot \boldsymbol{\epsilon}_{\mathbf{1}}=\left\langle\lambda_{3}, \lambda_{1}\right\rangle^{2}, \quad k_{2} \boldsymbol{b} \cdot \boldsymbol{\epsilon}_{\mathbf{2}}=\left\langle\lambda_{3}, \lambda_{2}\right\rangle^{2}, \\
& \boldsymbol{b} \cdot \boldsymbol{k}_{\mathbf{1}}=\left\langle\lambda_{3}, \lambda_{1}\right\rangle\left[\lambda_{3}, \bar{\lambda}_{1}\right]=\frac{\left\langle\lambda_{3}, \lambda_{1}\right\rangle\left\langle\lambda_{3}, \lambda_{2}\right\rangle}{\left\langle\lambda_{2}, \lambda_{1}\right\rangle}\left(k_{1}+k_{2}-k_{3}\right), \\
& \boldsymbol{b} \cdot \boldsymbol{k}_{\mathbf{2}}=\frac{\left\langle\lambda_{3}, \lambda_{1}\right\rangle\left\langle\lambda_{3}, \lambda_{2}\right\rangle}{\left\langle\lambda_{1}, \lambda_{2}\right\rangle}\left(k_{1}+k_{2}-k_{3}\right) .
\end{aligned}
$$


Adding (3.36) and (3.37) and substituting (3.40), we see that $\tilde{R}$ must satisfy the equation:

$$
\frac{1}{2}\left(\frac{\partial^{2} \tilde{R}}{\partial k_{1}^{2}}-\frac{\partial^{2} \tilde{R}}{\partial k_{2}^{2}}\right)-\frac{2}{k_{1}+k_{2}-k_{3}}\left(\frac{\partial \tilde{R}}{\partial k_{1}}-\frac{\partial \tilde{R}}{\partial k_{2}}\right)=\tilde{R}\left(\frac{1}{k_{1}^{2}}-\frac{1}{k_{2}^{2}}\right)
$$

$\boldsymbol{b} \propto \boldsymbol{\epsilon}_{1}:$ Now, let us choose $b_{\alpha \dot{\alpha}}=2 \lambda_{1 \alpha} \hat{\lambda}_{1 \dot{\alpha}}=2 k_{1} \epsilon_{1}$. We need the Ward identity term. In position space, this is given by (2.56) and its Fourier transform is given by (3.23). Using this, and keeping track of the factor of $\frac{1}{2}$ in (C.7), we see that (3.31) evaluates to:

$$
\begin{aligned}
W & =3 \frac{\left\langle\lambda_{1}, \lambda_{3}\right\rangle^{2}}{k_{3}^{5} k_{1} k_{2}}\left\langle\lambda_{3}, \lambda_{1}\right\rangle\left[\lambda_{3}, \bar{\lambda}_{1}\right]\left(k_{1}^{3}-k_{2}^{3}\right) \\
& =3 \frac{\left\langle\lambda_{3}, \lambda_{1}\right\rangle^{3}\left\langle\lambda_{3}, \lambda_{2}\right\rangle}{k_{3}^{5} k_{1} k_{2}\left\langle\lambda_{2}, \lambda_{1}\right\rangle}\left(k_{1}+k_{2}-k_{3}\right)\left(k_{1}^{3}-k_{2}^{3}\right) .
\end{aligned}
$$

Next we see that

$$
\begin{aligned}
& \boldsymbol{b} \cdot \boldsymbol{k}_{\mathbf{2}}=\frac{\left\langle\lambda_{1}, \lambda_{2}\right\rangle\left\langle\lambda_{1}, \lambda_{3}\right\rangle}{\left\langle\lambda_{3}, \lambda_{2}\right\rangle}\left(k_{3}+k_{2}-k_{1}\right)=-\boldsymbol{b} \cdot \boldsymbol{k}_{\mathbf{3}} \\
& b_{\alpha \dot{\alpha}} \lambda_{4}^{\alpha} \hat{\lambda}_{3}^{\dot{\alpha}}=-\left\langle\lambda_{1}, \lambda_{3}\right\rangle^{2}, \quad k_{2} \boldsymbol{b} \cdot \boldsymbol{\epsilon}_{\mathbf{2}}=\left\langle\lambda_{1}, \lambda_{2}\right\rangle^{2} .
\end{aligned}
$$

This leads to the following equation for $\tilde{R}$ :

$$
\begin{aligned}
& \frac{1}{2}\left(\frac{\partial^{2} \tilde{R}}{\partial k_{2}^{2}}-\frac{\partial^{2} \tilde{R}}{\partial k_{3}^{2}}\right)+\frac{2}{k_{2}+k_{3}-k_{1}}\left(\frac{\partial \tilde{R}}{\partial k_{2}}-\frac{\partial \tilde{R}}{\partial k_{3}}\right) \\
& =\frac{\tilde{R}}{k_{2}^{2}}+3 \frac{k_{1}+k_{2}-k_{3}}{k_{2}+k_{3}-k_{1}} \frac{\left(k_{1}^{3}-k_{2}^{3}\right)}{k_{3}^{5} k_{1} k_{2}} .
\end{aligned}
$$

$\boldsymbol{b} \propto \boldsymbol{\epsilon}_{\mathbf{2}}$ : We do not need to explicitly compute the term with $b \propto \epsilon_{2}$ after this, since that equation should be obtainable just by interchanging particles 1 and 2 . So, we can immediately see that we must have the equation:

$$
\begin{aligned}
& \frac{1}{2}\left(\frac{\partial^{2} \tilde{R}}{\partial k_{1}^{2}}-\frac{\partial^{2} \tilde{R}}{\partial k_{3}^{2}}\right)+\frac{2}{k_{1}+k_{3}-k_{2}}\left(\frac{\partial \tilde{R}}{\partial k_{1}}-\frac{\partial \tilde{R}}{\partial k_{3}}\right) \\
& =\frac{\tilde{R}}{k_{1}^{2}}+3 \frac{k_{2}+k_{1}-k_{3}}{k_{1}+k_{3}-k_{2}} \frac{\left(k_{2}^{3}-k_{1}^{3}\right)}{k_{3}^{5} k_{2} k_{1}} .
\end{aligned}
$$

It is more convenient to derive another homogeneous equation by combining (3.45) and (3.44). From these two, we get:

$$
\begin{aligned}
& \left(k_{2}+k_{3}-k_{1}\right) \frac{\partial^{2} \tilde{R}}{\partial k_{2}^{2}}+\left(k_{1}+k_{3}-k_{2}\right) \frac{\partial^{2} \tilde{R}}{\partial k_{1}^{2}}-2 k_{3} \frac{\partial^{2} \tilde{R}}{\partial k_{3}^{2}} \\
& +2\left(\frac{\partial \tilde{R}}{\partial k_{2}}+\frac{\partial \tilde{R}}{\partial k_{1}}-2 \frac{\partial \tilde{R}}{\partial k_{3}}\right)=\tilde{R}\left(\frac{k_{1}+k_{3}-k_{2}}{k_{1}^{2}}+\frac{k_{2}+k_{3}-k_{1}}{k_{2}^{2}}\right) .
\end{aligned}
$$




\subsection{Comparison with direct momentum space computations}

It is now useful to translate our notation back to that of the previous subsection and write down our final equations. Contracting (3.2) with the polarization tensor and using spinor identities to rewrite the answer in the form (3.29) leads to a relation between $\tilde{R}$ and $S$

$$
\tilde{R}=-\frac{\left(k_{1}+k_{2}-k_{3}\right)^{2}}{k_{1} k_{2} k_{3}^{3}} \frac{S}{2}
$$

(See Appendix C.5 for a derivation). From here, (3.41) tells us that $S$ must satisfy the equation:

$$
\left(\Theta\left(k_{1}\right)-\Theta\left(k_{2}\right)\right) S=0 .
$$

This is precisely what we would get by substituting $\boldsymbol{b}=\boldsymbol{\epsilon}_{\mathbf{3}}$ in (3.18).

We can also write (3.46) in terms of $S$ rather than $\tilde{R}$. When we do this, we find that $S$ must satisfy the equation:

$$
\begin{aligned}
& 8 k_{1} k_{2}\left[S-k_{1} \frac{\partial S}{\partial k_{1}}-k_{2} \frac{\partial S}{\partial k_{2}}-k_{3} \frac{\partial S}{\partial k_{3}}\right]+2 k_{1} k_{2} k_{3}\left(k_{1}+k_{2}-k_{3}\right) \Theta\left(k_{3}\right) S \\
& +k_{1} k_{2}\left(\left(k_{1}-k_{3}\right)^{2}-k_{2}^{2}\right) \Theta\left(k_{2}\right) S+k_{1} k_{2}\left(-k_{1}^{2}+\left(k_{2}-k_{3}\right)^{2}\right) \Theta\left(k_{1}\right) S=0 .
\end{aligned}
$$

If we now use the fact that $S$ has dimension 1 and also the equation (3.48), we find the remarkably simple equation:

$$
\left(\Theta\left(k_{1}\right)-\Theta\left(k_{3}\right)\right) S=0 .
$$

So (3.50) and (3.48) are our final homogeneous equations, which can also be obtained directly in momentum space. These are separate from the inhomogeneous equation (3.44). The inhomogeneous equation can also be shown to be equivalent to (3.21). By substituting (3.47) into (3.44), we find that

$$
\begin{aligned}
& 2 k_{1} k_{3}\left(3 k_{1}^{2}-3 k_{2}^{2}+2 k_{2} k_{3}+k_{3}^{2}\right) \frac{\partial S}{\partial k_{3}}+2 k_{3}^{2}\left(3 k_{1}^{2}+\left(k_{2}-k_{3}\right)^{2}\right) \frac{\partial S}{\partial k_{1}} \\
& +k_{3}^{2} k_{1}\left(k_{1}+k_{2}-k_{3}\right)\left(k_{1}-k_{2}+k_{3}\right)\left(-\frac{\partial^{2} S}{\partial k_{3}^{2}}+\frac{\partial^{2} S}{\partial k_{1}^{2}}\right)-4 k_{1}\left(3 k_{1}^{2}-3 k_{2}^{2}+k_{3}^{2}\right) S \\
& +12\left(k_{1}^{4}-k_{1} k_{2}^{3}\right)=0 .
\end{aligned}
$$

Now, if we write $\frac{\partial^{2} S}{\partial k_{3}^{2}}=\Theta\left(k_{3}\right) S+\frac{2}{k_{3}} \frac{\partial S}{\partial k_{3}}$, use the fact that $\left[\Theta\left(k_{3}\right)-\Theta\left(k_{1}\right)\right] S=0$, and collect the terms proportional to the different partial derivatives of $S$, we find that this reduces to:

$$
k_{3}\left(k_{1}^{2}-k_{2}^{2}+k_{3}^{2}\right) \frac{\partial S}{\partial k_{3}}+2 k_{3}^{2} k_{1} \frac{\partial S}{\partial k_{1}}-\left(3 k_{1}^{2}-3 k_{2}^{2}+k_{3}^{2}\right) S+3\left(k_{1}^{3}-k_{2}^{3}\right)=0 .
$$

Now, if we use the fact that

$$
\sum_{m} k_{m} \frac{\partial S}{\partial k_{m}}=S
$$


to substitute for $k_{3} \frac{\partial S}{\partial k_{3}}$ in the equation above, and also use (3.4) we find that

$$
-\left(\boldsymbol{k}_{\mathbf{1}} \cdot \boldsymbol{k}_{\mathbf{3}}\right) k_{2} \frac{\partial}{\partial k_{2}} S+\left(\boldsymbol{k}_{\mathbf{2}} \cdot \boldsymbol{k}_{\mathbf{3}}\right) k_{1} \frac{\partial}{\partial k_{1}} S-\left(k_{1}^{2}-k_{2}^{2}\right) S+\frac{3}{2}\left(k_{1}^{3}-k_{2}^{3}\right)=0 .
$$

This is exactly the same as (3.21), if we use (3.48).

This concludes our demonstration that the differential equations obtained in spinor helicity variables are the same as those obtained directly in momentum space.

\section{Solving the Conformal Constraints}

The three point correlator involving two scalar and one tensor perturbations was calculated for a model of inflation in [11]. The answer is given in equations (4.10) and (4.11) of [11] in terms of the function

$$
I=-\left(k_{1}+k_{2}+k_{3}\right)+\frac{\sum_{i>j} k_{i} k_{j}}{\left(k_{1}+k_{2}+k_{3}\right)}+\frac{k_{1} k_{2} k_{3}}{\left(k_{1}+k_{2}+k_{3}\right)^{2}} .
$$

From this result we can read off the functional form for the corresponding $\left\langle O O T_{i j}\right\rangle$ coefficient. This gives

$$
S=-I=-\left[-\left(k_{1}+k_{2}+k_{3}\right)+\frac{\sum_{i>j} k_{i} k_{j}}{\left(k_{1}+k_{2}+k_{3}\right)}+\frac{k_{1} k_{2} k_{3}}{\left(k_{1}+k_{2}+k_{3}\right)^{2}}\right] .
$$

It is easy to check that this function solves the three equations (3.21), (3.25), (3.26) above.

\subsection{Uniqueness}

In this subsection we will see that (4.2) is the unique solution to (3.24), (3.25), (3.26) which meets all the required conditions.

We begin by noting that the set of functions

$$
f_{z}(k)=(1+i k z) e^{-i k z}
$$

with $z$ allowed to range over both positive and negative values forms a complete set. Any function $\mathcal{H}(k)$ can be expanded in terms of this set,

$$
\mathcal{H}(k)=\int_{-\infty}^{\infty} \tilde{\phi}(z) f_{z}(k) d z .
$$

The point is that $\tilde{\phi}$ is a kind of souped up Fourier transform of $\mathcal{H}(k)$. Let $\phi(k)$ be the Fourier transform of $\tilde{\phi}(z)$. Then (4.4) gives

$$
\mathcal{H}(k)=\phi(k)-k \phi^{\prime}(k)=-k^{2} \frac{d}{d k}\left(\frac{\phi(k)}{k}\right),
$$

which can be solved to obtain

$$
\phi(k)=-k \int^{k} \frac{\mathcal{H}(x)}{x^{2}} d x,
$$


and correspondingly

$$
\tilde{\phi}(z)=\int_{-\infty}^{\infty}-\left[k e^{i k z} \int^{k} \frac{\mathcal{H}(x)}{x^{2}} d x\right] \frac{d k}{2 \pi} .
$$

Note that (4.6) determines $\phi(k)$ up to a term proportional to $k$ and this in turns leads to an ambiguity proportional to $\delta^{\prime}(z)$ in $\tilde{\phi}(z)$, but this ambiguity drops out of the integral in (4.4) leading to a well defined value for $\mathcal{H}(k)$.

Thus the most general solution can be expanded as

$$
\begin{aligned}
S\left(k_{1}, k_{2}, k_{3}\right)=\int[ & \left(1+i k_{1} z_{1}\right) e^{-i k_{1} z_{1}}\left(1+i k_{2} z_{2}\right) e^{-i k_{2} z_{2}} \\
& \left.\times\left(1+i k_{3} z_{3}\right) e^{-i k_{3} z_{3}} \mathcal{M}\left(z_{1}, z_{2}, z_{3}\right)\right] d z_{1} d z_{2} d z_{3},
\end{aligned}
$$

where each $z_{i}$ integral runs over $(-\infty, \infty)$.

Now note that since

$$
\Theta(k) f_{z}(k)=-z^{2} f_{z}(k),
$$

the functions $f_{z}(k)$ are eigenvectors of the operator $\Theta(k){ }^{8}$ It then follows that (3.25), (3.26), for $S$ given in (4.8) lead to the conditions

$$
z_{1}^{2}=z_{2}^{2}=z_{3}^{3}
$$

As a result an allowed solution can be written in the following form:

$$
S=\sum_{n_{1}, n_{2}, n_{3}= \pm 1} \int_{0}^{\infty} \mathcal{F}_{n_{1} n_{2} n 3}(z) \mathcal{M}_{n_{1} n_{2} n_{3}}(z) d z,
$$

where $\mathcal{M}_{n_{1}, n_{2}, n_{3}}$ are a set of 8 functions for the 8 possible combinations of $n_{1}, n_{2}, n_{3}$ and

$$
\mathcal{F}_{n_{1} n_{2} n_{3}}(z)=\left(1+i n_{1} k_{1} z\right) e^{-i n_{1} k_{1} z}\left(1+i n_{2} k_{2} z\right) e^{-i n_{2} k_{2} z}\left(1+i n_{3} k_{3} z\right) e^{-i n_{3} k_{3} z} .
$$

Next, we apply the dilatation constraint:

$$
\left(k_{1} \frac{\partial}{\partial k_{1}}+k_{2} \frac{\partial}{\partial k_{2}}+k_{3} \frac{\partial}{\partial k_{3}}\right) S=S .
$$

We notice that:

$$
\begin{aligned}
& \left(\frac{k_{1} \partial}{\partial k_{1}}+\frac{k_{2} \partial}{\partial k_{2}}+\frac{k_{3} \partial}{\partial k_{3}}\right) S-S=\sum_{n_{1}, n_{2}, n_{3}= \pm 1} \int_{0}^{\infty} \mathcal{M}_{n_{1} n_{2} n_{3}}(z)\left(z \frac{\partial}{\partial z}-1\right) \mathcal{F}_{n_{1} n_{2} n_{3}}(z) \\
& =-\sum_{n_{1}, n_{2}, n_{3}= \pm 1} \int_{0}^{\infty}\left(\frac{\partial}{\partial z} z+1\right) \mathcal{M}_{n_{1} n_{2} n_{3}}(z) \mathcal{F}_{n_{1} n_{2} n_{3}}(z) d z
\end{aligned}
$$

which leads to

$$
-\frac{\partial}{\partial z} z \mathcal{M}_{n_{1}, n_{2} n_{3}}(z)=\mathcal{M}_{n_{1}, n_{2}, n_{3}}(z)
$$

\footnotetext{
${ }^{8}$ The functions $f_{z}(k)$ are in fact solutions to the massless scalar equation in de Sitter space with $z$ being conformal time.
} 
This provides us with

$$
\mathcal{M}_{n_{1}, n_{2}, n_{3}}=\frac{m_{n_{1}, n_{2}, n_{3}}}{z^{2}}
$$

where $m_{n_{1}, n_{2}, n_{3}}$ is an arbitrary constant. Essentially all that we are saying that the $z$ dependence of $\mathcal{M}_{n_{1}, n_{2}, n_{3}}$ is fixed by noting that it must have dimension 2 and $z$ has dimension -1 .

In going from (4.14) to (4.15), we tacitly assumed that $\mathcal{M}$ was regular at the origin so that we could drop the boundary term at 0. However, the result in (4.17) makes (4.14) divergent both at 0 and at $\infty$. We can be more careful as follows. To define the integral at $z=\infty$, we can analytically continue the correlator to give the $k_{i}$ a small imaginary part. To define the integral at $z=0$, we can define it by:

$$
S=\left.\sum_{n_{1}, n_{2}, n_{3}= \pm 1} m_{n_{1} n_{2} n_{3}} \int_{0}^{\infty} \mathcal{F}_{n_{1} n_{2} n_{3}}(z) \frac{d z}{z^{2}} \equiv \sum_{n_{1}, n_{2}, n_{3}= \pm 1} m_{n_{1} n_{2} n_{3}} \int_{\epsilon}^{\infty} \mathcal{M}_{n_{1} n_{2} n_{3}}(z)\right|_{\epsilon^{0}},
$$

which means that we regulate the integral, by changing the range to $(\epsilon, \infty)$ and then pick up the $\epsilon^{0}$ term. This prescription now makes the resulting integral well defined while preserving its behaviour under scale transformations.

The prescription above leads to:

$$
\begin{aligned}
S=\sum_{n_{1}, n_{2}, n_{3}= \pm 1} m_{n_{1} n_{2} n_{3}}( & -n_{1} n_{2} n_{3} \frac{k_{2} k_{3} k_{1}}{\left(n_{1} k_{1}+n_{2} k_{2}+n_{3} k_{3}\right)^{2}}+n_{1} k_{1}+n_{2} k_{2}+n_{3} k_{3} \\
& \left.-\frac{n_{1} n_{2} k_{1} k_{2}+n_{2} n_{3} k_{3} k_{2}+n_{1} n_{3} k_{1} k_{3}}{n_{1} k_{1}+n_{2} k_{2}+n_{3} k_{3}}\right) .
\end{aligned}
$$

Actually there are only four distinct terms in the sum above since the function of $k_{i}$ 's within the bracket on the RHS above only changes by an overall sign when the sign of all three $n_{i}$ 's is changed. We can use this property to fix $n_{3}=+1$ so that $S$ is given by a sum over four terms

$$
\begin{gathered}
S=\sum_{n_{1}, n_{2}= \pm 1} m_{n_{1} n_{2}}\left(-n_{1} n_{2} \frac{k_{2} k_{3} k_{1}}{\left(n_{1} k_{1}+n_{2} k_{2}+k_{3}\right)^{2}}+n_{1} k_{1}+n_{2} k_{2}+k_{3}\right. \\
\left.-\frac{n_{1} n_{2} k_{1} k_{2}+n_{2} k_{3} k_{2}+n_{1} k_{1} k_{3}}{n_{1} k_{1}+n_{2} k_{2}+k_{3}}\right) .
\end{gathered}
$$

where $m_{n_{1}, n_{2}}=m_{n_{1} n_{2}+1}$.

So far we have used (3.25), (3.26). It is easy to show that the remaining equation (3.24) acting on the solution above gives rise to the two conditions

$$
\begin{aligned}
\sum_{n_{1}, n_{2}} m_{n_{1}, n_{2}} n_{1}^{3} & =1 \\
\sum_{n_{1}, n_{2}} m_{n_{1}, n_{2}} n_{2}^{3} & =1 .
\end{aligned}
$$




\subsection{Various Limits For The Momenta}

In this subsection we will show by considering two different limits for the momenta that one can rule out three of the four terms which appear in the sum in (4.20) leaving only the term with $n_{1}=n_{2}=1$. The normalization of this term is then fixed by (4.21),(4.22) leading to the unique result given in (4.2).

\subsubsection{First Limit}

First consider the limit where the momentum carried by the tensor perturbation is much smaller than that of the two scalar perturbations,

$$
k_{3} \ll k_{1} \simeq k_{2}
$$

In this limit the scalar perturbations can be taken to be propagating in an essentially constant metric $\gamma_{i j}$. The resulting wave function (2.25) can be calculated in two ways. Either by working directly with the boundary values, $\gamma_{i j}, \delta \phi$. Or by first taking a boundary metric which is flat, $\gamma_{i j}=\delta_{i j}$, and then transforming the answer by a coordinate transformation to the case of the constant metric $\gamma_{i j}$. The two answers must of course agree.

This gives rise to the condition, [11], that in this limit

$$
\left\langle T_{i j}\left(k_{3}\right) O\left(k_{1}\right) O\left(k_{2}\right)\right\rangle^{\prime} e^{s, i j}=-e^{s, i j} k_{2 i} k_{2 j} \frac{d}{d k_{2}^{2}}\left\langle O\left(k_{2}\right) O\left(-k_{2}\right)\right\rangle^{\prime},
$$

where the superscript prime on the two sides stands for the correlator without the factor of $(2 \pi)^{3} \delta^{3}\left(\sum \boldsymbol{k}_{\boldsymbol{i}}\right)$. From (A.5) and (3.27) this gives that in the limit (4.23)

$$
S \rightarrow \frac{3}{2} k_{2}
$$

One finds that this condition rules out the two terms in (4.20) where $n_{1}, n_{2}$ have the opposite sign so that

$$
\begin{aligned}
& S=\sum_{\left\{\left(n_{1}, n_{2}\right)=(+,+),\left(n_{1}, n_{2}\right)=(-,-)\right\}} m_{n_{1} n_{2}}\left(-n_{1} n_{2} \frac{k_{2} k_{3} k_{1}}{\left(n_{1} k_{1}+n_{2} k_{2}+k_{3}\right)^{2}}+n_{1} k_{1}+n_{2} k_{2}+k_{3}\right. \\
& \left.-\frac{n_{1} n_{2} k_{1} k_{2}+n_{2} k_{3} k_{2}+n_{1} k_{1} k_{3}}{n_{1} k_{1}+n_{2} k_{2}+k_{3}}\right) \text {. }
\end{aligned}
$$

\subsubsection{Second Limit and the OPE}

Next we examine the limit where $k_{2} \simeq k_{3} \gg k_{1}$. The behaviour in this limit is most easily understood if we can appeal to the operator product expansion (OPE). We have seen that the coefficient functions which appear in the wave function (2.23), (2.24), transform under the conformal symmetries like the correlation functions of a CFT. It is well known that in a CFT operators satisfy the operator product expansion. For the arguments that follow we will assume that this is true for the coefficient functions in the wave function as well. While this 
assumption is quite plausibly true we do not provide a proof for it here. ${ }^{9}$ In the next section we provide another argument for uniqueness that does not reply on the OPE.

To see how the argument goes let us first examine the limit which was studied above, where $k_{1}, k_{2}$ are large compared to $k_{3}$, but now using the OPE. We take

$$
\boldsymbol{k}_{2}=\boldsymbol{K}, \quad \boldsymbol{k}_{1}=-\boldsymbol{K}+\boldsymbol{k}_{3}, \quad \text { with } K \equiv|\boldsymbol{K}| \gg k_{3} .
$$

In position space we are considering the limit $\boldsymbol{x}_{1} \rightarrow \boldsymbol{x}_{\mathbf{2}}$ for the correlation function

$$
\left\langle O\left(\boldsymbol{x}_{1}\right) O\left(\boldsymbol{x}_{\mathbf{2}}\right) T_{\mu \nu}\left(\boldsymbol{x}_{\mathbf{3}}\right)\right\rangle .
$$

The operator product expansion tells us that in this limit the leading contribution comes from the term

$$
O(0) O(\boldsymbol{x})=\frac{x_{\mu} x_{\nu}}{x^{5}} T^{\mu \nu}(\boldsymbol{x})+\ldots
$$

where $\boldsymbol{x} \equiv \boldsymbol{x}_{2}-\boldsymbol{x}_{1}$.

The momentum space correlator is obtained by taking a Fourier transform of (4.28)

$$
\begin{aligned}
& \int\left\langle O\left(\boldsymbol{x}_{\mathbf{1}}\right) O\left(\boldsymbol{x}_{\mathbf{2}}\right) T^{\mu \nu}\left(\boldsymbol{x}_{\mathbf{3}}\right)\right\rangle e^{i\left(\left(\boldsymbol{k}_{\mathbf{1}} \cdot \boldsymbol{x}_{\mathbf{1}}\right)+\left(\boldsymbol{k}_{\mathbf{2}} \cdot \boldsymbol{x}_{\mathbf{2}}\right)+\left(\boldsymbol{k}_{\mathbf{3}} \cdot \boldsymbol{x}_{\mathbf{3}}\right)\right)} d^{3} x_{1} d^{3} x_{2} d^{3} x_{3} \\
& =\int\left\langle O(0) O\left(\boldsymbol{x}_{\mathbf{2}}-\boldsymbol{x}_{\mathbf{1}}\right) T^{\mu \nu}\left(\boldsymbol{x}_{\mathbf{3}}-\boldsymbol{x}_{\mathbf{1}}\right)\right\rangle e^{i\left(\left(\boldsymbol{k}_{\mathbf{1}}+\boldsymbol{k}_{\mathbf{2}}+\boldsymbol{k}_{\mathbf{3}}\right) \cdot \boldsymbol{x}_{\mathbf{1}}+\boldsymbol{k}_{\mathbf{2}} \cdot\left(\boldsymbol{x}_{\mathbf{2}}-\boldsymbol{x}_{\mathbf{1}}\right)+\boldsymbol{k}_{\mathbf{3}}\left(\boldsymbol{x}_{\mathbf{3}}-\boldsymbol{x}_{\mathbf{1}}\right)\right)} d^{3} x_{1} d^{3} x_{2} d^{3} x_{3} \\
& =(2 \pi)^{3} \delta^{3}\left(\boldsymbol{k}_{\mathbf{1}}+\boldsymbol{k}_{\mathbf{2}}+\boldsymbol{k}_{\mathbf{3}}\right) \int\left\langle O(0) O\left(\boldsymbol{x}_{\mathbf{2}}-\boldsymbol{x}_{\mathbf{1}}\right) T^{\mu \nu}\left(\boldsymbol{x}_{\mathbf{3}}-\boldsymbol{x}_{\mathbf{1}}\right)\right\rangle e^{i\left(\boldsymbol{k}_{2} \cdot\left(\boldsymbol{x}_{\mathbf{2}}-\boldsymbol{x}_{\mathbf{1}}\right)+\boldsymbol{k}_{\mathbf{3}} \cdot\left(\boldsymbol{x}_{\mathbf{3}}-\boldsymbol{x}_{\mathbf{1}}\right)\right)} d^{3} x_{2} d^{3} x_{3} .
\end{aligned}
$$

In the limit (4.27) it follows from (4.29) that the momentum space correlator should go like

$$
\int \frac{x^{\mu} x^{\nu}}{x^{5}} e^{i \boldsymbol{K} \cdot \boldsymbol{x}} d^{3} x \sim O\left(K^{0}\right)
$$

Since the expression (3.27) already has a factor of $K^{2}$ outside, we learn that

$$
S \sim \frac{k_{3}^{3}}{K^{2}}
$$

where we have inserted the correct factor of $k_{3}$ by dimensional analysis. It is easy to check that this only happens in the sum in (4.20) if $n_{1}, n_{2}$ have the same sign.

For example, consider the term in (4.20) with $n_{1}=n_{2}=1$. And scale $k_{3} \rightarrow \lambda k_{3}$ and expand in powers of $\lambda$, for small $\lambda$. We get:

$$
\begin{aligned}
-S & =\frac{3 k_{1}}{2}+\frac{3\left(\boldsymbol{k}_{\mathbf{1}} \cdot \boldsymbol{k}_{\mathbf{3}}\right) \lambda}{4 k_{1}}+\left(\frac{k_{3}{ }^{2}}{k_{1}}-\frac{\left(\boldsymbol{k}_{\mathbf{1}} \cdot \boldsymbol{k}_{\mathbf{3}}\right)^{2}}{4 k_{1}{ }^{3}}\right) \lambda^{2} \\
& +\lambda^{3}\left(\frac{3\left(\boldsymbol{k}_{\mathbf{1}} \cdot \boldsymbol{k}_{\mathbf{3}}\right)^{3}}{16 k_{1}{ }^{5}}-\frac{9 k_{3}{ }^{2}\left(\boldsymbol{k}_{\mathbf{1}} \cdot \boldsymbol{k}_{\mathbf{3}}\right)}{16 k_{1}{ }^{3}}-\frac{3 k_{3}{ }^{3}}{8{k_{1}}^{2}}\right) .
\end{aligned}
$$

\footnotetext{
${ }^{9}$ In the AdS/CFT correspondence which is related by analytic continuation to the dS case one can plausibly provide an argument for the operator product expansion from the bulk using the prescription for calculating the boundary correlation functions from the bulk, the properties of the bulk to boundary propagator, etc. By analytic continuation one would expect then to be able to show this for the coefficient functions in the dS case as well.
} 
One might naively believe that this contradicts (4.32). However, it is rather interesting that all the terms that grow too fast with $K$ are actually analytic in at least two momenta and so lead to contact terms when transformed to position space.

For example, we have

$$
\begin{aligned}
& \int k_{1 i} k_{2 j} \frac{3 k_{1}}{2} \delta^{3}\left(\boldsymbol{k}_{\mathbf{1}}+\boldsymbol{k}_{\mathbf{2}}+\boldsymbol{k}_{\mathbf{3}}\right) e^{-i\left(\boldsymbol{k}_{\mathbf{1}} \cdot \boldsymbol{x}_{\mathbf{1}}+\boldsymbol{k}_{\mathbf{2}} \cdot \boldsymbol{x}_{\mathbf{2}}+\boldsymbol{k}_{\mathbf{3}} \cdot \boldsymbol{x}_{\mathbf{3}}\right)} d^{3} k_{1} d^{3} k_{2} d^{3} k_{3} \\
& =-\frac{\partial}{\partial x_{1}^{i}} \frac{\partial}{\partial x_{2}^{j}} \int \frac{3 k_{1}}{2} e^{-i\left(\boldsymbol{k}_{\mathbf{1}} \cdot\left(\boldsymbol{x}_{\mathbf{1}}-\boldsymbol{x}_{\mathbf{2}}\right)+\boldsymbol{k}_{\mathbf{3}} \cdot\left(\boldsymbol{x}_{\mathbf{3}}-\boldsymbol{x}_{\mathbf{2}}\right)\right)} d^{3} k_{1} d^{3} k_{3} \\
& =-(2 \pi)^{3} \frac{\partial}{\partial x_{1}^{i}} \frac{\partial}{\partial x_{2}^{j}} \delta\left(\boldsymbol{x}_{3}-\boldsymbol{x}_{2}\right) \int \frac{3 k_{1}}{2} e^{-i\left(\boldsymbol{k}_{\mathbf{1}} \cdot\left(\boldsymbol{x}_{\mathbf{1}}-\boldsymbol{x}_{\mathbf{2}}\right)\right)} d^{3} k_{1}+\ldots
\end{aligned}
$$

where ... are subleading in $\lambda$.

The first non-analytic term in (4.33) is the term that goes like $\frac{k_{3}^{3}}{k_{1}^{2}}$, which is indeed of the form that we expected in (4.32)!

It is easy to check that if we consider a term in (4.20) where $n_{1}, n_{2}$ have opposite sign we will not get an answer consistent with the OPE. For example consider the term with $n_{1}=-1, n_{2}=n_{3}=1$, we have

$$
-S=\frac{1}{\lambda} \frac{2 k_{3} k_{1}^{4}+\left(\boldsymbol{k}_{\mathbf{1}} \cdot \boldsymbol{k}_{\mathbf{3}}\right) k_{1}^{3}}{\left(\left(\boldsymbol{k}_{\mathbf{1}} \cdot \boldsymbol{k}_{\mathbf{3}}\right)+k_{1} k_{3}\right)^{2}} .
$$

This is already non-analytic and is clearly of the wrong form.

Having considered the limit where $k_{1}, k_{2}$ are large compared to $k_{3}$ we can finally turn to the limit of interest where $k_{2}, k_{3}$ are large compared to $k_{1}$. In position space this corresponds to the case where $x_{2} \rightarrow x_{3}$, in which case we expect the dominant OPE

$$
\begin{aligned}
O\left(x_{2}\right) T_{\mu \nu}\left(x_{3}\right)= & A \frac{\left(x_{2}-x_{3}\right)_{\mu}\left(x_{2}-x_{3}\right)_{\nu}}{x_{2}-x_{3}^{5}} O\left(x_{3}\right)+B \frac{\left(x_{2}-x_{3}\right)_{\mu} \partial_{\nu}+\left(x_{2}-x_{3}\right)_{\nu} \partial_{\mu}}{x_{2}-x_{3}^{4}} O\left(x_{3}\right) \\
& +\frac{C}{x_{3}^{3}} \partial_{\mu} \partial_{\nu} O\left(x_{3}\right) .
\end{aligned}
$$

We are now concerned with the limit where $k_{3}=K, k_{2}=-K-k_{1}$ and $\frac{K}{k_{1}}$ is large. The terms that multiply $A$ and $B$ might seem like they scale like $K^{0}$ in this limit, but this is deceptive. In fact, if we work through the Fourier transform, we expect that these terms give rise to

$$
\frac{K_{\mu} K_{\nu}+K_{(\mu} k_{1 \nu}+k_{1 \mu} k_{1 \nu}}{K^{2}}
$$

in Fourier space. Of these terms only the last one $-k_{1 \mu} k_{1 \nu}$ is meaningful, since the others point along $K$, and yield 0 when contracted with a transverse polarization tensor for the stress-tensor. A similar logic applies for the term that multiplies $B$. So, in fact, all the three terms in (4.36) should give terms that scale like $\frac{1}{K^{2}}$ when transformed to momentum space.

This now implies that $S$ itself must scale like $\frac{1}{K^{2}}$ since the full correlator is given by $S$ multiplied with $e^{s, i j} k_{1 i} k_{2 j}(3.27)$, and even though $\boldsymbol{k}_{\mathbf{2}}=-\boldsymbol{K}-\boldsymbol{k}_{\mathbf{1}}$, since $e^{s, i j} K_{j}=0$, this 
factor scales like $O\left(K^{0}\right)$. It is now simple to see that of the two terms that remain in (4.26) the only one which gives the correct behaviour for $S$ is the one with $n_{1}=n_{2}=1$. The analysis of expanding the terms in this limit and comparing with the required behaviour is completely analogous to the one above and we will skip the details.

To summarize, by considering two limits for the momenta we learn that of the four terms which could have been present in $S$, (4.20) only one term survives giving the final result in (4.2).

\subsection{Another Argument for Uniqueness}

Now, we give a second argument — which does not assume the OPE — for why only the choice $n_{1}=1, n_{2}=1$ is allowed. This argument closely follows an argument made in [18]. We will show that any correlator that arises from a local interaction in de Sitter space, in the presence of the Bunch Davies boundary conditions described in section 2.3 will have a pole in the quantity $E=k_{1}+k_{2}+k_{3}$. Demanding the existence of this pole immediately tells us that the choice $n_{1}=n_{2}=1$ is the only one that is allowable.

The argument is as follows. The correlator that we are interested in is the coefficient of a particular term in the expansion of the wave-function of the Universe. First, let us consider the case where the correlator can be computed order by order in perturbation theory. Operatively, this means that we start with the solutions to the free equations of motion for the metric and the scalar perturbations, and then correct them perturbatively. ${ }^{10}$

The solutions to the free equations of motion are given by:

$$
\begin{aligned}
& \gamma_{i j}\left(\boldsymbol{k}_{\boldsymbol{3}}\right)=\kappa_{\gamma} e_{i j}^{s}\left(1-i \eta k_{3}\right) e^{i k_{3} \eta} e^{i \boldsymbol{k}_{\mathbf{3}} \cdot \boldsymbol{x}}, \\
& \delta \phi\left(\boldsymbol{k}_{\boldsymbol{n}}\right)=\kappa_{\phi}\left(1-i \eta k_{n}\right) e^{i k_{n} \eta} e^{i \boldsymbol{k}_{\boldsymbol{n}} \cdot \boldsymbol{x}},
\end{aligned}
$$

where $\kappa_{\gamma}$ and $\kappa_{\phi}$ are some constants and $\boldsymbol{k}_{\boldsymbol{n}}$ may be either $\boldsymbol{k}_{\mathbf{1}}$ or $\boldsymbol{k}_{\mathbf{2}}$. Note that, as we explained above, our choice of boundary conditions in the far past fixes the sign of the exponent involving $\eta$.

Without making any assumptions about the form of the interaction, the leading contribution to the correlator in such an expansion will be given by acting with some linear functional (which comes from the interaction vertices in the action (2.1)) on these perturbations. Denoting this linear functional by $S_{\text {int }}$, we have

$$
\left\langle T^{i j}\left(\boldsymbol{k}_{\mathbf{3}}\right) O\left(\boldsymbol{k}_{\mathbf{1}}\right) O\left(\boldsymbol{k}_{\mathbf{2}}\right)\right\rangle=S_{\mathrm{int}}\left[\gamma_{i j}\left(\boldsymbol{k}_{\mathbf{3}}\right), \delta \phi\left(\boldsymbol{k}_{\mathbf{1}}\right), \delta \phi\left(\boldsymbol{k}_{\mathbf{2}}\right)\right] .
$$

Let us focus on the contribution to the correlator that comes from very early times i.e. from $\eta \rightarrow-\infty$. While, (2.1) may be very complicated with many higher derivative terms, in this limit the variation (4.39) is controlled by the highest power of $\eta$ that appears:

$$
\left\langle T^{i j}\left(\boldsymbol{k}_{\mathbf{3}}\right) O\left(\boldsymbol{k}_{\mathbf{1}}\right) O\left(\boldsymbol{k}_{\mathbf{2}}\right)\right\rangle=\int d \eta \eta^{m} e^{i \eta\left(k_{1}+k_{2}+k_{3}\right)} \mathcal{C}_{f}^{i j}\left(\boldsymbol{k}_{\mathbf{1}}, \boldsymbol{k}_{\mathbf{2}}, \boldsymbol{k}_{\mathbf{3}}\right)+\ldots,
$$

\footnotetext{
${ }^{10}$ This is almost a universal approach to perturbation theory. For example, even in the Vasiliev theory, which involves an infinite number of derivatives, this is precisely how correlation functions are calculated. (See [19], sections 4.2 and 4.3.)
} 
where the ... indicate terms that come with lower powers of $\eta$ and $\mathcal{C}^{f}$ is independent of $\eta$. We get a contribution of $\eta^{-4}$ from the $\sqrt{-g}$ in the action. The $\eta^{m}$ arises by combining this term with the terms linear in $\eta$ in (4.38) and other factors of $\eta$ from the inverse metric. We can easily check that the two-derivative interaction already gives rise to $m=1$ and since higher derivatives require more factors of the unperturbed inverse metric, they give rise to higher powers of $\eta$. Evidently, doing this integral leads to

$$
\left\langle T^{i j}\left(\boldsymbol{k}_{\mathbf{3}}\right) O\left(\boldsymbol{k}_{\mathbf{1}}\right) O\left(\boldsymbol{k}_{\mathbf{2}}\right)\right\rangle=\frac{\Gamma[m+1]}{E^{m+1}} \mathcal{C}_{f}^{i j}\left(\boldsymbol{k}_{\mathbf{1}}, \boldsymbol{k}_{\mathbf{2}}, \boldsymbol{k}_{\mathbf{3}}\right)+\ldots,
$$

where $\ldots$ are now terms that have lower order poles in $E$.

With only a small amount of additional work, we can actually show that $\mathcal{C}_{f}^{i j}$ is related to the flat space scattering amplitude for two scalars and a graviton. The reader can already see this from the answer written in the form (3.29). We refer the reader to [18] (see section 5) for details on why we should have expected this.

Returning to the problem at hand we see that since it is only $n_{1}=n_{2}=1$ that gives the correct pole, this is the only allowed choice. This proves the uniqueness of our solution.

At this point, the reader may wonder why higher derivative terms, which would have given rise to higher poles, do not contribute in this answer. This is related to the fact that the form of the three-point on-shell amplitude for two scalars and a graviton in four flat dimensions is exact, already at tree-level, and is not altered either by higher-derivatives or loop corrections.

It is quite simple to see this, since there is a unique Lorentz invariant that can be formed from the physical quantities at hand - the polarization tensor of the graviton, and the three four-dimensional momenta $\boldsymbol{k}_{\mathbf{1}}, \boldsymbol{k}_{\mathbf{2}}, \boldsymbol{k}_{\mathbf{3}}$. The on-shell condition tells us that $\boldsymbol{k}_{\mathbf{1}}{ }^{2}=\boldsymbol{k}_{\mathbf{2}}{ }^{2}=\boldsymbol{k}_{\mathbf{3}}{ }^{2}=$ 0 and since $\boldsymbol{k}_{\mathbf{1}}+\boldsymbol{k}_{\mathbf{2}}+\boldsymbol{k}_{\mathbf{3}}=0$, all dot products between the momenta vanish as well. ${ }^{11}$ So, the only four dimensional Lorentz invariant we can form is $e_{i j}^{s} k_{1}^{i} k_{2}^{j}$. All that loop corrections or higher derivatives could possibly do is to renormalize the coefficient of this quantity but this is also fixed by the Ward identities.

It is this fact about flat-space scattering amplitudes that is related to the uniqueness of our correlation function.

\subsection{Final Solution}

As mentioned above the unique solution for $S$ was obtained above in (4.2). The overall normalization followed from the use of the normalization of the two point function $\left\langle O\left(k_{1}\right) O\left(k_{2}\right)\right\rangle$ given in (3.22) which in turn determined the Ward identity (3.23).

Instead as discussed in Appendix $\mathrm{A}$ it is convenient to take the two point function $\left\langle O\left(k_{1}\right) O\left(k_{2}\right)\right\rangle$ to be normalized as given in (A.5) so that its normalization differs from (3.22)

\footnotetext{
${ }^{11}$ The momenta themselves do not have to be collinear if we allow them to be complex.
} 
by a factor of ${ }^{12} c$. With this choice the solution for the correlator becomes

$$
\left\langle O\left(k_{1}\right) O\left(k_{2}\right) T_{i j}\left(k_{3}\right)\right\rangle e^{s, i j}=-2(2 \pi)^{3} c \delta\left(\sum_{i} \boldsymbol{k}_{i}\right) e^{s, i j} k_{1 i} k_{2 j} S .
$$

From the general arguments of section 2 this should be the value for the coefficient function, $\left\langle O O T_{i j}\right\rangle e^{s, i j}$, in the wave function (2.23).

\section{Final Result}

Using the wave function (2.23) and (2.21) it is now a simple matter to find the three point correlator involving two scalar perturbations $\zeta\left(\boldsymbol{k}_{1}\right), \zeta\left(\boldsymbol{k}_{2}\right)$ and one tensor perturbation $\gamma_{i j}\left(\boldsymbol{k}_{3}\right)$ with polarization $e^{s, i j}$.

One finds that it is given by

$$
\left\langle\zeta\left(k_{1}\right) \zeta\left(k_{2}\right) \gamma_{s}\left(k_{3}\right)\right\rangle=(2 \pi)^{3} \delta\left(\sum_{i} \boldsymbol{k}_{i}\right) \frac{1}{\Pi_{i}\left(2 k_{i}^{3}\right)}\left(\frac{4 H^{4}}{M_{p l}^{4} c}\right)\left(\frac{H^{2}}{\dot{\phi}^{2}}\right) e^{s, i j} k_{1 i} k_{2 j} S\left(k_{1}, k_{2}, k_{3}\right),
$$

with

$$
S\left(k_{1}, k_{2}, k_{3}\right)=\left(k_{1}+k_{2}+k_{3}\right)-\frac{\sum_{i>j} k_{i} k_{j}}{\left(k_{1}+k_{2}+k_{3}\right)}-\frac{k_{1} k_{2} k_{3}}{\left(k_{1}+k_{2}+k_{3}\right)^{2}} .
$$

In this formula $\dot{\phi}$ is the time derivative of the inflaton and $c$ is a constant which is defined from the normalization of the scalar two-point function given in (A.5). This constant can be set to unity by rescaling $\dot{\phi}$. When the two derivative approximation is valid, in the normalization where $c=1, \dot{\phi}$ is related to the slow roll parameter $\epsilon$ by $(2.10) . \gamma_{s}$ is related to the tensor perturbation by

$$
\gamma_{i j}\left(k_{3}\right)=\gamma_{s}\left(k_{3}\right) e_{i j}^{s}\left(k_{3}\right)
$$

where $e_{i j}^{s}\left(k_{3}\right)$ is the polarization which is transverse and traceless, (3.13), with normalization given in (A.3).

Equation (5.1) is the main result of this paper.

By comparing this result with the two point functions for the scalar and tensor perturbations given in (A.5), (A.6) of the appendix A we see that the normalization of the correlator is completely fixed in terms of the normalization of these two two-point functions.

For conventional slow-roll inflation the answer above agrees, up to an overall sign, with that obtained in [11], with $c=1$ and $\phi$ being the canonically normalized inflaton.

\section{Conclusions}

In this paper we have studied the three point function involving two scalars and one tensor perturbation. We showed that this correlator is completely fixed by the $S O(1,4)$ symmetries of

\footnotetext{
${ }^{12}$ The constant $c$ can be set to unity by rescaling the inflaton, but keeping it explicit allows for the normalization of the inflaton to be determined in an independent manner.
} 
de Sitter space, up to small corrections. Our final result is given in (5.1). The normalizations for the scalar and tensor two point functions are given in (A.5) and (A.6); we see that the normalization of the three point function is fixed in terms of the normalization of the two point functions.

Our result is based on three main assumptions. First, that the inflationary dynamicsincluding the scalar sector-approximately preserves the full $S O(1,4)$ conformal group of isometries of de Sitter space. Second, that there is only one scalar field during inflation. And third, that the initial state is the Bunch-Davies vacuum. Other than these assumptions the result is general and essentially model independent. In particular it should apply to models where higher derivative corrections in gravity are important, as was discussed in the introduction.

The general nature of this result means that this three point function is observationally a good way to test if the inflationary dynamics had the full conformal group including the special conformal transformations as its symmetries. It is worth emphasizing that the two point functions do not by themselves allow for a test of this feature. In conventional slow-roll inflation there is one relation between the various parameters which arises as follows. The tensor two point function allows for a determination of $H^{2} / M_{P l}^{2}$ from its normalization and for $\epsilon$, defined in (2.5), from its tilt. The normalization of the scalar two-point function goes like $\frac{H^{2}}{M_{P l}^{2}} \frac{H^{2}}{\dot{\phi}^{2}}$ and is then fixed since $\frac{\dot{\phi}}{H}$ is determined in terms of $\epsilon$ by (2.10). However, once higher derivative corrections are included (2.10) need not be valid any longer even when the full conformal group is approximately preserved. For example (2.7) could receive corrections due to higher powers of curvature becoming important in the action (2.1). Thus this relation between the parameters of the two point functions does not allow us to test whether the special conformal transformations were good symmetries during inflation.

Corrections to our result for the three point function will arise from effects which break the $S O(1,4)$ symmetries. These can be of two kinds. Effects which break the special conformal symmetries but preserve scale invariance and effects which break scale invariance. Examples of the breaking of special conformal invariance include a speed of sound which is different from unity. More generally, these effects can be parameterized using the effective Lagrangian approach discussed in [7]. The breaking of scale invariance occurs because the Hubble constant and the inflaton slowly evolve during inflation and are not constant. When the momenta of the three perturbations in the correlator are of the same order of magnitude one immediate way to incorporate some of the resulting corrections is to set the parameters, $H, \dot{\phi}$ which enter in (5.1), to take their values at the time of horizon crossing for the modes. ${ }^{13}$ More generally, corrections due to the breaking of scale invariance are of order the slow roll parameters and about $1 \%$ in order of magnitude.

As stated above our result applies to models of single field inflation. When more than one scalar is present both adiabatic and isocurvature perturbations can be present and it

\footnotetext{
${ }^{13}$ In the squeezed limit, when one momentum is much smaller one can also incorporate similar effects by carrying out an analysis along the lines of section 4.2 .
} 
is harder to come up with model independent results. We can always still go to the gauge where $\zeta=0$, discussed for the single scalar case in 2.1.2. And then work in a basis where the scalar field perturbations, $\delta \phi_{i}, i=1, \cdots N$, have a diagonal two point function. Assuming that scalars are approximately massless we get the two-point functions to be ${ }^{14}$

$$
\left\langle\delta \phi_{i}\left(\boldsymbol{k}_{1}\right) \delta \phi_{j}\left(\boldsymbol{k}_{2}\right)\right\rangle=\delta_{i j}(2 \pi)^{3} \delta^{3}\left(\boldsymbol{k}_{\mathbf{1}}+\boldsymbol{k}_{\mathbf{2}}\right) \frac{H^{2}}{M_{p l}^{2}} \frac{1}{2} \frac{1}{k_{1}^{3}} .
$$

The three point functions for the scalar and tensor perturbations then easily follows and is again diagonal in this basis of scalar perturbations and takes the model independent form (5.1) (with $c=1$ ). The model dependence in the result enters when we try to obtain the three point function in terms of the the curvature perturbation, $\zeta$, which is defined for all time and conserved after the modes cross the horizon. The value of this perturbation and its correlations depend on how the various scalars affect the end of inflation and this is model dependent.

The analysis in this paper is based on earlier papers $[11,13]$. In [13] it was shown that working in the de Sitter approximation the three point tensor perturbation can be significantly constrained from symmetry considerations alone. Unlike tensor perturbations when dealing with scalars the small breaking of de Sitter symmetries in the inflationary background cannot be totally ignored. However for the correlation function of interest in this paper this breaking can be incorporated, at least to leading order in the slow-roll parameters, in a straightforward manner. As explained in section 2 one first works in the gauge where $\zeta=0$ and calculates the correlation function in terms of the scalar perturbation $\delta \phi$, then transforms to the gauge where the $\zeta \neq 0$ using (2.21). The calculation in terms of $\delta \phi$ can be done in de Sitter space and the breaking of de Sitter invariance enters only in the last step through the factor of $\frac{\dot{\phi}}{H}$ in (2.21). This is analogous to using the relation (2.33) in conformal perturbation theory and computing the correlation function in terms of the scalar operator $O$ in the CFT.

It is important to try to extend this analysis to other correlation functions especially the three point scalar correlator which is observationally most significant. The analysis is more complicated here since in general one cannot get away by simply taking the breaking of the de Sitter symmetries into account in the manner described in the previous paragraph. This can be seen from the results for the conventional slow-roll case in [11] where it was found that the scalar three-point function is suppressed by an additional factor of $\sqrt{\epsilon}$ leading to an answer that goes like ${ }^{15} \frac{H^{4}}{M_{P l}^{4} \epsilon}$. Despite these complications, it would be worthwhile to consider a CFT which has say just the stress tensor and a scalar as its low dimension operators and ask how much the scalar correlators are constrained by CFT considerations alone along the lines of $[20]$.

\footnotetext{
${ }^{14}$ Here we have rescaled $\delta \phi$ to set a possible constant $c$ which appears in the normalization on the RHS to unity.

${ }^{15}$ This fact also follows from CFT by noting that the three-point function of an exactly marginal operator must vanish.
} 
We have used both scale and special conformal invariance in deriving our result. We have already discussed the possibility that the scalar sector could break the special conformal symmetries badly. On the gravity side translations, rotations and scale invariance uniquely lead to de Sitter space, which is then also invariant under special conformal transformations. However, more generally, when higher spin fields are also excited it is conceivable that one has time dependent solutions with translations, rotations and scale invariance symmetry but without special conformal invariance. It would be worth developing an understanding of such solutions and their possible role in the early Universe. ${ }^{16}$ The correlator studied here could be used to distinguish solutions of this type also from de Sitter space.

\section{Acknowledgments}

SPT thanks Yoskie Sumitomo for early collaboration. We are grateful to N. Iizuka, E. Komatsu, S. Kachru, J. Maldacena, G. Mandal, S. Minwalla, T. Souradeep and A. Yadav for discussions. IM thanks the organizers of the ICTS school on cosmology and gravity waves, Dec. 2011. SR and SPT thank the organizers of the "strings discussion meeting" at ICTS (Bangalore) for a stimulating conference where some of this research was carried out. SR acknowledges the support of a Ramanujan Fellowship from the Department of Science and Technology, Govt. of India. SR is grateful to Brown University, where part of this work was carried out, for its hospitality. SPT acknowledges the support of a J.C. Bose Fellowship from the Department. of Science and Technology, Govt. of India. Most of all, we would all like to acknowledge our extensive debt to the people of India.

\section{A The Two Point Function and Normalizations}

In this appendix we discuss the two point function and related issues about normalizations of correlation functions. The wave function at quadratic order can be read off from (2.24)

$$
\begin{aligned}
\psi=\exp \left(\frac{M_{p l}^{2}}{H^{2}}[\right. & -\frac{1}{2} \int \frac{d^{3} k}{(2 \pi)^{3}} \frac{d^{3} k^{\prime}}{(2 \pi)^{3}} \delta \phi(\boldsymbol{k}) \delta \phi\left(\boldsymbol{k}^{\prime}\right)\left\langle O(-\boldsymbol{k}) O\left(-\boldsymbol{k}^{\prime}\right)\right\rangle \\
& \left.\left.-\frac{1}{2} \int \frac{d^{3} k}{(2 \pi)^{3}} \frac{d^{3} k^{\prime}}{(2 \pi)^{3}} \gamma_{s}(\boldsymbol{k}) \gamma_{s^{\prime}}\left(\boldsymbol{k}^{\prime}\right)\left\langle T^{s}(-\boldsymbol{k}) T^{s^{\prime}}\left(-\boldsymbol{k}^{\prime}\right)\right\rangle\right]\right) .
\end{aligned}
$$

Here the labels $s, s^{\prime}$ denote the two polarizations of the graviton. In our notation a graviton can be written as a linear combination of its two polarizations

$$
\gamma_{i j}(\boldsymbol{k})=\sum_{s=1,2} \gamma_{s} e_{i j}^{s}(\boldsymbol{k})
$$

where the polarization tensors are normalized so that

$$
e^{s, i j} e_{i j}^{s^{\prime}}=2 \delta^{s, s^{\prime}}
$$

\footnotetext{
${ }^{16}$ For a review of higher spin fields and related issues see [21].
} 
For the stress energy tensor we define

$$
T^{s}(\boldsymbol{k}) \equiv T_{i j}(\boldsymbol{k}) e^{s, i j}(-\boldsymbol{k}) .
$$

Translational and rotational invariance along with scaling symmetry fixes the form of the two point functions to be

$$
\begin{aligned}
\left\langle O\left(\boldsymbol{k}_{\mathbf{1}}\right) O\left(\boldsymbol{k}_{\mathbf{2}}\right)\right\rangle & =c k_{1}^{3}(2 \pi)^{3} \delta^{3}\left(\boldsymbol{k}_{\mathbf{1}}+\boldsymbol{k}_{\mathbf{2}}\right), \\
\left\langle T^{s}\left(\boldsymbol{k}_{\mathbf{1}}\right) T^{s^{\prime}}\left(\boldsymbol{k}_{\mathbf{2}}\right)\right\rangle & =k_{1}^{3}(2 \pi)^{3} \delta^{3}\left(\boldsymbol{k}_{\mathbf{1}}+\boldsymbol{k}_{\mathbf{2}}\right)\left(\frac{\delta^{s s^{\prime}}}{2}\right) .
\end{aligned}
$$

A constant could have appeared on the RHS of (A.6) but that can be absorbed into a redefinition of $H$. The constant $c$ which appears on the RHS of (A.5) could also have been set to unity by rescaling the operator $O$. However doing so also requires us to rescale the inflaton perturbation $\delta \phi$ which is the source for $O$. It is convenient instead to not do this rescaling and keep the constant $c$ explicit in (A.5).

Substituting (A.5), (A.6) in the wave function one can easily show that the resulting two-point functions for the perturbations are

$$
\begin{aligned}
& \left\langle\delta \phi\left(\boldsymbol{k}_{\mathbf{1}}\right) \delta \phi\left(\boldsymbol{k}_{\mathbf{2}}\right)\right\rangle=(2 \pi)^{3} \delta^{3}\left(\boldsymbol{k}_{\mathbf{1}}+\boldsymbol{k}_{\mathbf{2}}\right) \frac{H^{2}}{M_{p l}^{2}} \frac{1}{2 c} \frac{1}{k_{1}^{3}}, \\
& \left\langle\gamma_{s}\left(\boldsymbol{k}_{\mathbf{1}}\right) \gamma_{s^{\prime}}\left(\boldsymbol{k}_{\mathbf{2}}\right)\right\rangle=(2 \pi)^{3} \delta\left(\boldsymbol{k}_{\mathbf{1}}+\boldsymbol{k}_{\mathbf{2}}\right) \frac{H^{2}}{M_{p l}^{2}} \frac{1}{2 k_{1}^{3}}\left(2 \delta_{s, s^{\prime}}\right) .
\end{aligned}
$$

Using (2.21) we get from (A.7) that the two point function of the scalar perturbation is

$$
\left\langle\zeta\left(\boldsymbol{k}_{\mathbf{1}}\right) \zeta\left(\boldsymbol{k}_{\mathbf{2}}\right)\right\rangle=(2 \pi)^{3} \delta^{3}\left(\boldsymbol{k}_{\mathbf{1}}+\boldsymbol{k}_{\mathbf{2}}\right) \frac{H^{2}}{M_{p l}^{2}} \frac{1}{2 c} \frac{H^{2}}{\dot{\phi}^{2}} \frac{1}{k_{1}^{3}} .
$$

(A.8), (A.9) agree with the results of the standard slow-roll two -derivative theory when $c=1$ and $\phi$ is the canonically normalized inflaton. More generally $c$ can be set to unity by rescaling $\phi$.

\section{B Details of the Equations for Special Conformal Invariance}

From (3.2) and (3.3) we learn that

$$
\begin{aligned}
M_{i j}\left(\boldsymbol{k}_{1}, \boldsymbol{k}_{2}, \boldsymbol{k}_{3}\right)= & k_{1 i} k_{1 j} f_{1}\left(k_{1}, k_{2}, k_{3}\right)+k_{2 i} k_{2 j} f_{1}\left(k_{2}, k_{1}, k_{3}\right) \\
& +\left(k_{1 i} k_{2 j}+k_{2 i} k_{1 j}\right) f_{2}\left(k_{1}, k_{2}, k_{3}\right)+\delta_{i j} f_{3}\left(k_{1}, k_{2}, k_{3}\right) .
\end{aligned}
$$

Multiplying by $k_{3 i}\left(k_{1 j}-\frac{k_{3 j}\left(k_{1} \cdot k_{3}\right)}{k_{3}^{2}}\right)$ we get

$k_{3 i}\left(k_{1 j}-\frac{k_{3 j}\left(k_{1} \cdot k_{3}\right)}{k_{3}^{2}}\right) M_{i j}\left(\boldsymbol{k}_{1}, \boldsymbol{k}_{2}, \boldsymbol{k}_{3}\right)=\left[k_{1}^{2}-\frac{\left(\boldsymbol{k}_{\mathbf{1}} \cdot \boldsymbol{k}_{\mathbf{3}}\right)^{2}}{k_{3}^{2}}\right]\left(\left(\boldsymbol{k}_{\mathbf{1}} \cdot \boldsymbol{k}_{\mathbf{3}}\right)\left(f_{1}-f_{2}\right)+\left(\boldsymbol{k}_{\mathbf{2}} \cdot \boldsymbol{k}_{\mathbf{3}}\right)\left(f_{2}-f_{1}^{T}\right)\right)$. 
Now, choosing $\boldsymbol{b} \propto \boldsymbol{k}_{1}-\frac{\boldsymbol{k}_{3}\left(\boldsymbol{k}_{1} \cdot \boldsymbol{k}_{3}\right)}{k_{3}^{2}}$ in $(3.18)$ we get

$$
\begin{gathered}
4 k_{1 i} k_{1 j} e^{s, i j}\left[\left(1+k_{1} \frac{\partial}{\partial k_{1}}\right) f_{1}-\left(1+k_{2} \frac{\partial}{\partial k_{2}}\right) f_{1}^{T}+\left(k_{2} \frac{\partial}{\partial k_{2}}-k_{1} \frac{\partial}{\partial k_{1}}\right) f_{2}\right. \\
\left.-\frac{\left(\boldsymbol{k}_{\mathbf{1}} \cdot \boldsymbol{k}_{\mathbf{3}}\right)}{k_{3}} \frac{\partial}{\partial k_{3}}\left(f_{1}-f_{2}\right)+\frac{\left(\boldsymbol{k}_{\mathbf{3}} \cdot \boldsymbol{k}_{\mathbf{2}}\right)}{k_{3}} \frac{\partial}{\partial k_{3}}\left(f_{1}^{T}-f_{2}\right)\right] \\
+k_{1 i} k_{2 j} e^{s, i j}\left[k_{1}^{2}-\frac{\left(\boldsymbol{k}_{\mathbf{1}} \cdot \boldsymbol{k}_{\mathbf{3}}\right)}{k_{3}^{2}}\right]\left(\Theta\left(k_{1}\right)-\Theta\left(k_{2}\right)\right)\left(2 f_{2}-f_{1}-f_{1}^{T}\right)=0 .
\end{gathered}
$$

Using $k_{2 j} e^{s, i j}=-\left(k_{1 j}+k_{3 j}\right) e^{s, i j}=-k_{1 j} e^{s, i j},($ B.3) reduces to

$$
\begin{aligned}
e^{s, i j} k_{1 i} k_{1 j}\{ & 4\left[\left(1+k_{1} \frac{\partial}{\partial k_{1}}\right) f_{1}-\left(1+k_{2} \frac{\partial}{\partial k_{2}}\right) f_{1}^{T}+\left(k_{2} \frac{\partial}{\partial k_{2}}-k_{1} \frac{\partial}{\partial k_{1}}\right) f_{2}\right. \\
& \left.-\frac{\left(\boldsymbol{k}_{\mathbf{1}} \cdot \boldsymbol{k}_{\mathbf{3}}\right)}{k_{3}} \frac{\partial}{\partial k_{3}}\left(f_{1}-f_{2}\right)+\frac{\left(\boldsymbol{k}_{\mathbf{2}} \cdot \boldsymbol{k}_{\mathbf{3}}\right)}{k_{3}} \frac{\partial}{\partial k_{3}}\left(f_{1}^{T}-f_{2}\right)\right] \\
& \left.-\left(k_{1}^{2}-\frac{\left(\boldsymbol{k}_{\mathbf{1}} \cdot \boldsymbol{k}_{\mathbf{3}}\right)^{2}}{k_{3}^{2}}\right)\left(\Theta\left(k_{1}\right)-\Theta\left(k_{2}\right)\right)\left(2 f_{2}-f_{1}-f_{1}^{T}\right)\right\}=0
\end{aligned}
$$

Since the polarization can be chosen so that $e^{s, i j} k_{1 i} k_{1 j}$ does not vanish the quantity within the curly brackets must vanish leading to

$$
\begin{aligned}
4[ & \left(k_{1} \frac{\partial}{\partial k_{1}}-\frac{\left(\boldsymbol{k}_{\mathbf{1}} \cdot \boldsymbol{k}_{\mathbf{3}}\right)}{k_{3}} \frac{\partial}{\partial k_{3}}\right)\left(f_{1}-f_{2}\right)-\left(k_{2} \frac{\partial}{\partial k_{2}}-\frac{\left(\boldsymbol{k}_{\mathbf{2}} \cdot \boldsymbol{k}_{\mathbf{3}}\right)}{k_{3}} \frac{\partial}{\partial k_{3}}\right)\left(f_{1}^{T}-f_{2}\right) \\
& \left.+\left(f_{1}-f_{2}\right)-\left(f_{1}^{T}-f_{2}\right)\right]-\left(k_{1}^{2}-\frac{\left(\boldsymbol{k}_{\mathbf{1}} \cdot \boldsymbol{k}_{\mathbf{3}}\right)^{2}}{k_{3}^{2}}\right)\left(\Theta\left(k_{1}\right)-\Theta\left(k_{2}\right)\right)\left(2 f_{2}-f_{1}-f_{1}^{T}\right)=0 .
\end{aligned}
$$

In terms of $S \equiv\left[\left(f_{1}-f_{2}\right)+\left(f_{1}^{T}-f_{2}\right)\right] / 2$ and $A \equiv\left[\left(f_{1}-f_{2}\right)-\left(f_{1}^{T}-f_{2}\right)\right] / 2$ this becomes

$$
\begin{aligned}
& 4\left[\left(k_{1} \frac{\partial}{\partial k_{1}}-\frac{\boldsymbol{k}_{1} \cdot \boldsymbol{k}_{3}}{k_{3}} \frac{\partial}{\partial k_{3}}\right)(S+A)-\left(k_{2} \frac{\partial}{\partial k_{2}}-\frac{\boldsymbol{k}_{2} \cdot \boldsymbol{k}_{3}}{k_{3}} \frac{\partial}{\partial k_{3}}\right)(S-A)+2 A\right] \\
& -2\left(k_{1}^{2}-\frac{\left(\boldsymbol{k}_{1} \cdot \boldsymbol{k}_{3}\right)^{2}}{k_{3}^{2}}\right)\left(\Theta\left(k_{1}\right)-\Theta\left(k_{2}\right)\right) S=0 .
\end{aligned}
$$

Similarly, (B.2) in terms of $S, A$ becomes,

$k_{3 i}\left(k_{1 j}-\frac{k_{3 j}\left(\boldsymbol{k}_{\mathbf{1}} \cdot \boldsymbol{k}_{\mathbf{3}}\right)}{k_{3}^{2}}\right) M_{i j}\left(\boldsymbol{k}_{1}, \boldsymbol{k}_{2}, \boldsymbol{k}_{3}\right)=\left[k_{1}^{2}-\frac{\left(\boldsymbol{k}_{\mathbf{1}} \cdot \boldsymbol{k}_{\mathbf{3}}\right)^{2}}{k_{3}^{2}}\right]\left(\left(\boldsymbol{k}_{\mathbf{1}} \cdot \boldsymbol{k}_{\mathbf{3}}\right)(S+A)-\left(\boldsymbol{k}_{\mathbf{2}} \cdot \boldsymbol{k}_{\mathbf{3}}\right)(S-A)\right)$,

which can be used to solve for $A$ and gives

$$
A=\frac{\left(\boldsymbol{k}_{\mathbf{1}}-\boldsymbol{k}_{\mathbf{2}}\right) \cdot \boldsymbol{k}_{\mathbf{3}}}{k_{3}^{2}} S-\frac{M_{i j} k_{3 i} \epsilon_{\perp j}}{k_{3}^{2} \epsilon_{\perp}^{2}}
$$


where $\epsilon_{\perp j} \equiv k_{1 j}-\frac{k_{3 j}\left(\boldsymbol{k}_{1} \cdot \boldsymbol{k}_{3}\right)}{k_{3}^{2}}$. (We caution the reader that this is different from the null transverse vector $\epsilon_{3}$ that has appeared above.) Substituting in (B.6) this leads to

$$
\begin{aligned}
4[ & \left(\frac{\boldsymbol{k}_{2} \cdot \boldsymbol{k}_{3}}{k_{3}^{2}} k_{1} \frac{\partial}{\partial k_{1}}-\frac{\boldsymbol{k}_{1} \cdot \boldsymbol{k}_{3}}{k_{3}^{2}} k_{2} \frac{\partial}{\partial k_{2}}\right) S+\frac{\boldsymbol{k}_{3} \cdot\left(\boldsymbol{k}_{2}-\boldsymbol{k}_{1}\right)}{k_{3}^{2}} S \\
& \left.+\left(k_{1} \frac{\partial}{\partial k_{1}}+k_{2} \frac{\partial}{\partial k_{2}}+k_{3} \frac{\partial}{\partial k_{3}}\right)\left(\frac{M_{i j} k_{3 i} \epsilon_{\perp j}}{2 k_{3}^{2} \epsilon_{\perp}^{2}}\right)+\frac{M_{i j} k_{3 i} \epsilon_{\perp j}}{k_{3}^{2} \epsilon_{\perp}^{2}}\right] \\
& +\left(k_{1}^{2}-\frac{\left(\boldsymbol{k}_{1} \cdot \boldsymbol{k}_{3}\right)^{2}}{k_{3}^{2}}\right)\left(\Theta\left(k_{1}\right)-\Theta\left(k_{2}\right)\right) S=0 .
\end{aligned}
$$

Next, using the Ward identity, (3.23) we get

$$
\frac{M_{i j} k_{3 i} \epsilon_{\perp j}}{\epsilon_{\perp}^{2}}=-k_{1}^{3}+k_{2}^{3} .
$$

Substituting (B.10) in (B.9) after some algebra gives (3.21).

Finally we consider taking $\boldsymbol{b}$ to be orthogonal to all $\boldsymbol{k}_{i}$ so that $\boldsymbol{b} \cdot \boldsymbol{k}=0$. We can also choose a polarization so that $b_{i} k_{1 j} e^{s, i j} \neq 0$. (3.18) then gives

$$
\begin{aligned}
& \left(1+k_{1} \partial_{k_{1}}\right) f_{1}-\left(1+k_{2} \partial_{k_{2}}\right) f_{1}^{T}+\left(k_{2} \partial_{k_{2}}-k_{1} \partial_{k_{1}}\right) f_{2}-\frac{\left(\boldsymbol{k}_{\mathbf{3}} \cdot \boldsymbol{k}_{\mathbf{1}}\right)}{k_{3}} \partial_{k_{3}}\left(f_{1}-f_{2}\right) \\
& +\frac{\left(\boldsymbol{k}_{\mathbf{3}} \cdot \boldsymbol{k}_{\mathbf{2}}\right)}{k_{3}} \partial_{k_{3}}\left(f_{1}^{T}-f_{2}\right)=0 .
\end{aligned}
$$

The reader will notice that the LHS above is the first two lines of the LHS of (B.4). Thus the analysis above when applied to (B.11) directly leads to (3.24).

\section{Spinor Helicity Formalism}

In this section we provide some further details on the calculations of subsection 3.2. We will use the spinor helicity formalism that was first introduced in [13] although our notation is similar to that of [17]. The paper [17] analyzed conformal field theory correlators in a Lorentzian spacetime; here our correlators obey the constraints of conformal invariance in a Euclidean spacetime. However, most of the formalism carries over directly as we show below.

\section{C.1 Notation}

Given a Euclidean 3 -momentum $\boldsymbol{k}=\left(k_{1}, k_{2}, k_{3}\right)$, we convert it into spinors using

$$
k_{\alpha \dot{\alpha}}=k \sigma_{\alpha \dot{\alpha}}^{0}+k_{1} \sigma_{\alpha \dot{\alpha}}^{1}+k_{2} \sigma_{\alpha \dot{\alpha}}^{2}+k_{3} \sigma_{\alpha \dot{\alpha}}^{3}=\lambda_{\alpha} \bar{\lambda}_{\dot{\alpha}}
$$

where

$$
|\boldsymbol{k}| \equiv \sqrt{\boldsymbol{k} \cdot \boldsymbol{k}}=\sqrt{k_{1}^{2}+k_{2}^{2}+k_{3}^{2}}
$$

We can raise and lower spinor indices using the $\epsilon$ tensor. We choose the $\epsilon$ tensor to be $i \sigma_{2}$ for both the dotted and the undotted indices. This means that

$$
\epsilon^{01}=1=-\epsilon^{10},
$$


and spinor dot products are defined via

$$
\left\langle\lambda_{1}, \lambda_{2}\right\rangle=\epsilon^{\alpha \beta} \lambda_{1 \alpha} \lambda_{2 \beta}=\lambda_{1 \alpha} \lambda_{2}^{\alpha}, \quad\left\langle\bar{\lambda}_{1}, \bar{\lambda}_{2}\right\rangle=\epsilon^{\dot{\alpha} \dot{\beta}} \bar{\lambda}_{1 \dot{\alpha}} \bar{\lambda}_{2 \dot{\beta}}=\bar{\lambda}_{1 \dot{\alpha}} \bar{\lambda}_{2}^{\dot{\alpha}}
$$

In the case of four-dimensional flat-space scattering amplitudes, all expressions can be written in terms of the two kinds of dot products above. However, in our case, we should expect our expressions for $\mathrm{CFT}_{3}$ correlators to only have a manifest $S O(3)$ invariance. This means that we might have mixed products between dotted and undotted indices. Such a mixed product extracts the $z$-component of vector and is performed by contracting with $\sigma^{0}$

$$
2|\boldsymbol{k}|=\left(\sigma^{0}\right)^{\alpha \dot{\alpha}} k_{\alpha \dot{\alpha}} \equiv[\lambda, \bar{\lambda}] \text {. }
$$

The reader should note that we use square brackets only for this mixed product; products of both left and right handed spinors are denoted by angular brackets. Second, we note that this mixed dot product is symmetric:

$$
[\lambda, \bar{\lambda}]=[\bar{\lambda}, \lambda]
$$

When we take the dot products of two 3-momenta, we have

$$
\begin{aligned}
& \boldsymbol{k} \cdot \boldsymbol{q} \equiv\left(k_{1} q_{1}+k_{2} q_{2}+k_{3} q_{3}\right) \\
& =-\frac{1}{2}\left(\left\langle\lambda_{k}, \lambda_{q}\right\rangle\left\langle\bar{\lambda}_{k}, \bar{\lambda}_{q}\right\rangle-\frac{1}{2}\left[\lambda_{k}, \bar{\lambda}_{k}\right]\left[\lambda_{q}, \bar{\lambda}_{q}\right]\right) .
\end{aligned}
$$

Another fact to keep in mind is that

$$
\begin{aligned}
& \boldsymbol{k}_{1}+\boldsymbol{k}_{2}=\boldsymbol{k}_{3} \\
& \Rightarrow \lambda_{1} \bar{\lambda}_{1}+\lambda_{2} \bar{\lambda}_{2}=\lambda_{3} \bar{\lambda}_{3}+\frac{1}{2}\left(\left[\lambda_{1}, \bar{\lambda}_{1}\right]+\left[\lambda_{2}, \bar{\lambda}_{2}\right]-\left[\lambda_{3}, \bar{\lambda}_{3}\right]\right) \sigma^{0} .
\end{aligned}
$$

We also need a way to convert dotted to undotted indices. We write

$$
\hat{\lambda}_{\dot{\alpha}}=-\sigma_{\alpha \dot{\alpha}}^{0} \lambda^{\alpha}, \quad \hat{\bar{\lambda}}_{\alpha}=-\sigma_{\alpha \dot{\alpha}}^{0} \bar{\lambda}^{\dot{\alpha}} .
$$

This has the property that

$$
\langle\bar{\mu}, \hat{\lambda}\rangle=[\bar{\mu}, \lambda]
$$

where the quantity on the right hand side is defined in (C.5).

With all this, we can write down polarization vectors for conserved currents. The polarization vectors for a momentum vector $\boldsymbol{k}$ associated with spinors $\lambda, \bar{\lambda}$ are given by

$$
\begin{aligned}
& \epsilon_{\alpha \dot{\alpha}}^{+}=2 \frac{\hat{\bar{\lambda}}_{\alpha} \bar{\lambda}_{\dot{\alpha}}}{[\lambda, \bar{\lambda}]}=\frac{\hat{\bar{\lambda}}_{\alpha} \bar{\lambda}_{\dot{\alpha}}}{k}, \\
& \epsilon_{\alpha \dot{\alpha}}^{-}=2 \frac{\lambda_{\alpha} \hat{\lambda}_{\dot{\alpha}}}{[\lambda, \bar{\lambda}]}=\frac{\lambda_{\alpha} \hat{\lambda}_{\dot{\alpha}}}{k} .
\end{aligned}
$$


These vectors are normalized so that

$$
\epsilon^{+} \cdot \epsilon^{+}=\epsilon^{-} \cdot \epsilon^{-}=0, \quad \epsilon^{+} \cdot \epsilon^{-}=2 .
$$

Polarization tensors for the stress tensor are just outer-products of these vectors with themselves:

$$
e_{i j}^{ \pm}=\epsilon_{i}^{ \pm} \epsilon_{j}^{ \pm}
$$

We again caution the reader that these are normalized differently from the $e_{i j}^{s}$ tensors, which appeared previously. These $e_{i j}^{ \pm}$tensors are linear combinations of those that correspond to "circularly polarized" gravitons.

\section{C.2 Conformal generators in momentum space}

As a prelude to understanding the action of conformal generators using spinor helicity variables, we need expressions for the conformal generators in momentum space. In position space we have $[22]$

$$
\begin{aligned}
D & =-i x^{i} \frac{\partial}{\partial x^{i}}-i \Delta, \\
K_{i} & =-2 i x_{i} \Delta-x^{j} S_{i j}-2 i x_{i} x^{j} \partial_{j}+i x^{2} \partial_{i} .
\end{aligned}
$$

When we Fourier transform this, we should replace $x^{i} \rightarrow i \frac{\partial}{\partial k_{i}}$ and $\frac{\partial}{\partial x^{i}} \rightarrow i k_{i}$. These replacements lead to

$$
\begin{aligned}
D & =i \frac{\partial}{\partial k_{i}} k_{i}-i \Delta=i(d-\Delta)+i k_{i} \frac{\partial}{\partial k_{i}} \\
K_{i} & =2 \Delta \frac{\partial}{\partial k^{i}}-i S_{i j} \frac{\partial}{\partial k_{j}}-2 \frac{\partial}{\partial k^{i}} \frac{\partial}{\partial k_{j}} k_{j}+\frac{\partial}{\partial k^{j}} \frac{\partial}{\partial k_{j}} k_{i} \\
& =2 \Delta \frac{\partial}{\partial k^{i}}-i S_{i j} \frac{\partial}{\partial k_{j}}-2 k_{j} \frac{\partial}{\partial k^{i}} \frac{\partial}{\partial k_{j}}+k_{i} \frac{\partial}{\partial k^{j}} \frac{\partial}{\partial k_{j}}-2(d+1-1) \frac{\partial}{\partial k^{i}} \\
& =2(\Delta-d) \frac{\partial}{\partial k^{i}}-i S_{i j} \frac{\partial}{\partial k_{j}}-2 k_{j} \frac{\partial}{\partial k^{i}} \frac{\partial}{\partial k_{j}}+k_{i} \frac{\partial}{\partial k^{j}} \frac{\partial}{\partial k_{j}} .
\end{aligned}
$$

The $D$ above should be distinguished from the $\tilde{D}$ in (3.6). For scalars, this can be recast as:

$$
\begin{aligned}
K_{i}^{s} & =2(\Delta-d) \frac{k_{i}}{k} \frac{\partial}{\partial k}-2 k_{j} \frac{\partial}{\partial k^{i}} \frac{k^{j}}{k} \frac{\partial}{\partial k}+k_{i} \frac{\partial}{\partial k^{j}} \frac{k^{j}}{k} \frac{\partial}{\partial k} \\
& =2(\Delta-d) \frac{k_{i}}{k} \frac{\partial}{\partial k}-2 \frac{k_{i}}{k} \frac{\partial}{\partial k}-2 k_{i} k \frac{\partial}{\partial k} \frac{1}{k} \frac{\partial}{\partial k}+\frac{d k_{i}}{k} \frac{\partial}{\partial k}+k_{i} k \frac{\partial}{\partial k} \frac{1}{k} \frac{\partial}{\partial k} \\
& =(2 \Delta-d-1) \frac{k_{i}}{k} \frac{\partial}{\partial k}-k_{i} \frac{\partial^{2}}{\partial k^{2}} .
\end{aligned}
$$

Here, we have just systematically replaced momentum derivatives using

$$
\frac{\partial}{\partial k_{i}}=\frac{\partial k}{\partial k_{i}} \frac{\partial}{\partial k}=\frac{k^{i}}{k} \frac{\partial}{\partial k}
$$

which is true for functions that depend only on $k$. 


\section{C.3 Conformal generators in spinor helicity variables for scalars}

Now we analyze how the double derivative operator $\frac{\partial}{\partial \lambda_{\alpha}} \frac{\partial}{\partial \lambda_{\dot{\alpha}}}$ can be used like the generator of special conformal transformations. Consider the object

$$
\tilde{K}_{i}=2 \frac{\partial}{\partial \lambda_{\alpha}} \frac{\partial}{\partial \bar{\lambda}_{\dot{\alpha}}} \sigma_{i \alpha \dot{\alpha}}
$$

We can convert these derivatives to momentum derivatives. Recall that we have

$$
\lambda_{\beta} \bar{\lambda}_{\dot{\beta}}=k_{m} \sigma_{\beta \dot{\beta}}^{m}+k \sigma_{\beta \dot{\beta}}^{0},
$$

and also,

$$
k_{j}=\frac{1}{2} \lambda_{\alpha} \lambda_{\dot{\alpha}} \bar{\sigma}_{j}^{\dot{\alpha} \alpha}
$$

This allows us to convert the spinorial derivatives to momentum derivatives as follows.

$$
\begin{aligned}
& \sigma_{i \alpha \dot{\alpha}} \frac{\partial}{\partial \lambda_{\alpha}} \frac{\partial}{\partial \bar{\lambda}_{\dot{\alpha}}}=\sigma_{i \alpha \dot{\alpha}} \frac{\partial}{\partial \lambda_{\alpha}} \frac{\partial k_{j}}{\partial \bar{\lambda}_{\dot{\alpha}}} \frac{\partial}{\partial k_{j}} \\
& =\sigma_{i \alpha \dot{\alpha}} \frac{\partial}{\partial \lambda_{\alpha}} \frac{1}{2} \bar{\sigma}_{j}^{\dot{\alpha} \beta} \lambda_{\beta} \frac{\partial}{\partial k_{j}}=\frac{1}{2} \sigma_{i \alpha \dot{\alpha}}\left(\bar{\sigma}_{j}^{\dot{\alpha} \alpha} \frac{\partial}{\partial k_{j}}+\frac{1}{2} \bar{\sigma}_{j}^{\dot{\alpha} \beta} \lambda_{\beta} \bar{\sigma}_{k}^{\dot{\beta} \alpha} \bar{\lambda}_{\dot{\beta}} \frac{\partial}{\partial k_{k}} \frac{\partial}{\partial k_{j}}\right) .
\end{aligned}
$$

In this expression, it is important that the spacetime indices on $\sigma$ are summed only over $(1,2,3)$ i.e they are not summed over the 0-direction.

Now, we note that

$$
\begin{aligned}
& \sigma_{i \alpha \dot{\alpha}} \bar{\sigma}_{j}^{\dot{\alpha} \alpha}=2 \eta_{i j} \\
& \sigma_{i \alpha \dot{\alpha}} \bar{\sigma}_{j}^{\dot{\alpha} \beta} \sigma_{m \beta \dot{\beta}} \bar{\sigma}_{k}^{\dot{\beta} \alpha}=2\left(\eta_{i j} \eta_{k m}+\eta_{i k} \eta_{j m}-\eta_{i m} \eta_{k j}+i \epsilon_{i j k m}\right) .
\end{aligned}
$$

The totally antisymmetric term is not important since our expression is symmetric in $j$ and $k$. Second, note that the term involving $\sigma^{0}$ in (C.19) drops out since the expression above involves a trace over products of $\sigma$ matrices and none of the other $\sigma$ matrices take the value $\sigma^{0}$ and, as we have already noted, the $\epsilon$-tensor term is unimportant.

Using all this, we find that

$$
\tilde{K}_{i}=2 \frac{\partial}{\partial k^{i}}+2 k_{j} \frac{\partial}{\partial k_{j}} \frac{\partial}{\partial k^{i}}-k_{i} \frac{\partial}{\partial k_{j}} \frac{\partial}{\partial k^{j}} .
$$

We see that $\tilde{K}_{i}$ agrees with the form (C.15) where $\Delta=d-1$. (Up to an overall minus sign.)

Using (C.16), for $\Delta=2$ and $d=3$ this is the statement that

$$
2 b_{i} \sigma_{\alpha \dot{\alpha}}^{i} \frac{\partial}{\partial \lambda_{\alpha}} \frac{\partial}{\partial \bar{\lambda}_{\dot{\alpha}}}=(\boldsymbol{b} \cdot \boldsymbol{k}) \frac{\partial}{\partial k^{2}} .
$$

Now, consider a marginal scalar - $O$. (This has dimension $d$ ). We note that

$$
2 b_{i} \sigma_{\alpha \dot{\alpha}}^{i} \frac{\partial}{\partial \lambda_{\alpha}} \frac{\partial}{\partial \bar{\lambda}_{\dot{\alpha}}} \frac{O}{k}=(\boldsymbol{b} \cdot \boldsymbol{k}) \frac{\partial}{\partial k^{2}} \frac{O}{k}=(\boldsymbol{b} \cdot \boldsymbol{k})\left(\frac{1}{k} \frac{\partial^{2} O}{\partial k^{2}}-\frac{2}{k^{2}} \frac{\partial O}{\partial k}+\frac{2 O}{k^{3}}\right) .
$$


Comparing with (C.16), this means that

$$
2 b_{i} \sigma_{\alpha \dot{\alpha}}^{i} \frac{\partial}{\partial \lambda_{\alpha}} \frac{\partial}{\partial \bar{\lambda}_{\dot{\alpha}}} \frac{O}{k}=\frac{1}{k}\left(-\boldsymbol{b} \cdot \boldsymbol{K}^{\boldsymbol{s}}\right) O+(\boldsymbol{b} \cdot \boldsymbol{k}) \frac{2 O}{k^{3}} .
$$

Equation (C.26) tells us that if we act with the double derivative on a marginal scalar, divided by the appropriate power of $k$, we will still get a term on the right hand side. This is similar to the "Ward identity" term we get below for stress tensors, except here we find that the right hand side is proportional to the original correlator itself.

\section{C.4 Special conformal transformations on stress tensors in spinor-helicity vari- ables}

We would now like to determine how the double derivative acts on tensors contracted with polarization vectors.

Using (C.13), we write the polarization tensor as:

$$
e_{i j}^{-}=\frac{1}{k^{2}} \bar{\sigma}_{i}^{a \dot{a}} \bar{\sigma}_{j}^{b \dot{b}} \lambda_{a} \hat{\lambda}_{\dot{a}} \lambda_{b} \hat{\lambda}^{\dot{b}}
$$

Here, as opposed to [17], we are also being careful to denote sigma matrices with indices raised with a bar. This is simply a matter of convenience.

We would like to calculate:

$$
2 b_{k} \sigma_{\alpha \dot{\alpha}}^{k} \frac{\partial}{\partial \lambda_{\alpha}} \frac{\partial}{\partial \bar{\lambda}_{\dot{\alpha}}} \frac{e_{i j}^{-}}{k^{p}} T^{i j}
$$

where $p$ is a power of the momentum that we will fix for convenience later. It is convenient to define

$$
\tilde{e}_{i j}^{-} \equiv k^{2} e_{i j}^{-} ; \tilde{T}^{i j} \equiv \frac{T^{i j}}{k^{p+2}}
$$

and instead compute:

$$
2 b_{k} \sigma_{\alpha \dot{\alpha}}^{k} \frac{\partial}{\partial \lambda_{\alpha}} \frac{\partial}{\partial \bar{\lambda}_{\dot{\alpha}}} \tilde{e}_{i j}^{-} \tilde{T}^{i j}=2 b_{k} \sigma_{\alpha \dot{\alpha}}^{k}\left(\tilde{e}_{i j}^{-} \frac{\partial}{\partial \lambda_{\alpha}} \frac{\partial}{\partial \bar{\lambda}_{\dot{\alpha}}} \tilde{T}^{i j}+\left(\frac{\partial}{\partial \lambda_{\alpha}} \tilde{e}_{i j}^{-}\right) \frac{\partial}{\partial \bar{\lambda}_{\dot{\alpha}}} \tilde{T}^{i j}\right) .
$$

Here we have used the fact that $\tilde{e}_{i j}^{-}$has no dependence on $\bar{\lambda}$. Let us parse the various terms in this expression. We have

$$
\frac{\partial}{\partial \lambda_{\alpha}} \tilde{e}_{i j}^{-}=\left(\hat{\lambda}_{\dot{a}} \lambda_{b} \hat{\lambda}_{\dot{b}} \bar{\sigma}_{i}^{\alpha \dot{a}} \bar{\sigma}_{j}^{b \dot{b}}-\lambda_{a} \sigma_{\dot{a} \beta}^{0} \epsilon^{\alpha \beta} \lambda_{b} \hat{\lambda}_{\dot{b}} \bar{\sigma}_{i}^{a \dot{a}} \bar{\sigma}_{j}^{b \dot{b}}\right)+(i \leftrightarrow j)
$$

and

$$
\frac{\partial \tilde{T}^{i j}}{\partial \bar{\lambda}_{\dot{\alpha}}}=\frac{1}{2} \frac{\partial \tilde{T}^{i j}}{\partial k_{m}} \bar{\sigma}_{m}^{\beta \dot{\alpha}} \lambda_{\beta}
$$

When we put the two equations above together, we encounter the term

$$
b_{k} \sigma_{\alpha \dot{\alpha}}^{k} \hat{\lambda}_{\dot{a}} \lambda_{b} \hat{\lambda}_{\dot{b}} \bar{\sigma}_{i}^{\alpha \dot{a}} \bar{\sigma}_{j}^{b \dot{b}} \frac{\partial \tilde{T}^{i j}}{\partial k_{m}} \bar{\sigma}_{m}^{\beta \dot{\alpha}} \lambda_{\beta}=b_{m} \tilde{e}_{i j}^{-} \frac{\partial \tilde{T}^{i j}}{\partial k_{m}}+b_{i} \tilde{e}_{m j}^{-} \frac{\partial \tilde{T}^{i j}}{\partial k_{m}}-b_{k} \tilde{e}_{k j}^{-} \frac{\partial \tilde{T}^{i j}}{\partial k^{i}}
$$


where we have used the identity (See 2.43 in [23])

$$
\begin{aligned}
& \sigma^{\mu} \bar{\sigma}^{\nu} \sigma^{\rho}=\eta^{\mu \nu} \sigma^{\rho}-\eta^{\mu \rho} \sigma^{\nu}+\eta^{\nu \rho} \sigma^{\mu}+i \epsilon^{\mu \nu \rho \kappa} \sigma_{\kappa}, \\
& \bar{\sigma}^{\mu} \sigma^{\nu} \bar{\sigma}^{\rho}=\eta^{\mu \nu} \bar{\sigma}^{\rho}-\eta^{\mu \rho} \bar{\sigma}^{\nu}+\eta^{\nu \rho} \bar{\sigma}^{\mu}-i \epsilon^{\mu \nu \rho \kappa} \bar{\sigma}_{\kappa},
\end{aligned}
$$

and noticed that the last $\epsilon$ does not contribute since all indices are summed only over three dimensions here. With a few index gymnastics we can check that, also

$$
-b_{k} \sigma_{\alpha \dot{\alpha}}^{k} \lambda_{a} \sigma_{\dot{a} \gamma}^{0} \epsilon^{\alpha \gamma} \lambda_{b} \hat{\lambda}_{\dot{b}} \bar{\sigma}_{i}^{a \dot{a}} \bar{\sigma}_{j}^{b \dot{b}} \frac{\partial \tilde{T}^{i j}}{\partial k_{m}} \bar{\sigma}_{m}^{\beta \dot{\alpha}} \lambda_{\beta}=b_{m} \tilde{e}_{i j}^{-} \frac{\partial \tilde{T}^{i j}}{\partial k_{m}}+b_{i} \tilde{e}_{m j}^{-} \frac{\partial \tilde{T}^{i j}}{\partial k_{m}}-b_{k} \tilde{e}_{k j}^{-} \frac{\partial \tilde{T}^{i j}}{\partial k^{i}} .
$$

Putting together (C.33),(C.35), (C.31) in (C.30) (and using the fact that $k^{i} e_{i j}^{-}=0$ ) we find that

$$
\begin{aligned}
b^{k} \tilde{K}_{k}\left(e_{i j}^{-} \frac{T^{i j}}{k^{p}}\right) & =2\left[\frac{-(p+2)(\boldsymbol{b} \cdot \boldsymbol{k}) e_{i j}^{-} T^{i j}+(p+2) b^{k} e_{k j}^{-} k_{i} T^{i j}}{k^{p+2}}+b_{m} \frac{e_{i j}^{-}}{k^{p}} \frac{\partial T^{i j}}{\partial k_{m}}\right. \\
& \left.+b_{i} \frac{e_{m j}^{-}}{k^{p}} \frac{\partial T^{i j}}{\partial k_{m}}-b^{k} \frac{e_{k j}^{-}}{k^{p}} \frac{\partial T^{i j}}{\partial k^{i}}\right]+(i \leftrightarrow j)-\tilde{e}_{i j}^{-} b^{k} \tilde{K}_{k} \tilde{T}^{i j} .
\end{aligned}
$$

The factor of 2 works out by realizing that (C.33) and (C.35) give the same contribution, and that the factor of $\frac{1}{2}$ in (C.32) cancels with the factor of 2 in the definition of $\tilde{K}$.

Now, we are almost done. We just need to convert the action of $k$-derivatives on $\tilde{T}$ to the action of these derivatives on $T$. We see that

$$
\begin{aligned}
\frac{\partial}{\partial k_{m}} \tilde{T}^{i j} & =\frac{-(p+2)}{k^{p+4}} k^{m} T^{i j}+\frac{1}{k^{p+2}} \frac{\partial T^{i j}}{\partial k_{m}}, \\
\frac{\partial}{\partial k_{n}} \frac{\partial}{\partial k_{m}} \tilde{T}^{i j} & =(p+2)\left[\frac{(p+4)}{k^{p+6}} k^{n} k^{m} T^{i j}-\frac{\eta^{m n}}{k^{p+4}} T^{i j}-\frac{k^{m}}{k^{p+4}} \frac{\partial T^{i j}}{\partial k_{n}}-\frac{k^{n}}{k^{p+4}} \frac{\partial T^{i j}}{\partial k_{m}}\right] \\
& +\frac{1}{k^{p+2}} \frac{\partial^{2} T^{i j}}{\partial k_{n} \partial k_{m}}, \\
2 k_{n} \frac{\partial}{\partial k_{n}} \frac{\partial}{\partial k_{m}} \tilde{T}^{i j} & =2(p+2)\left[\frac{(p+3) k^{m}}{k^{p+4}} T^{i j}-\frac{k^{m} k_{n}}{k^{p+4}} \frac{\partial T^{i j}}{\partial k_{n}}-\frac{1}{k^{p+2}} \frac{\partial T^{i j}}{\partial k_{m}}\right] \\
& +\frac{2 k_{n}}{k^{p+2}} \frac{\partial^{2} T^{i j}}{\partial k_{n} \partial k_{m}} \\
-k_{m} \frac{\partial}{\partial k^{n}} \frac{\partial}{\partial k_{n}} \tilde{T}^{i j} & =-(p+2)\left[\frac{(p+4-d) k_{m}}{k^{p+4}} T^{i j}-\frac{2 k_{m} k_{n}}{k^{p+4}} \frac{\partial T^{i j}}{\partial k_{n}}\right]-\frac{k_{m}}{k^{p+2}} \frac{\partial^{2} T^{i j}}{\partial k_{n} \partial k^{n}} .
\end{aligned}
$$

All of these lead to

$$
\tilde{K}_{m} \tilde{T}^{i j}=(p+2)(p+d) \frac{k_{m}}{k^{p+2}} T^{i j}-\frac{2(p+1)}{k^{p+2}} \frac{\partial T^{i j}}{\partial k_{m}}+\frac{1}{k^{p+2}} \tilde{K}_{m} T^{i j} .
$$


Finally, putting together (C.36) and (C.38), and using $d=3$, we see that

$$
\begin{aligned}
& b^{k} \tilde{K}_{k}\left(e_{i j}^{-} \frac{T^{i j}}{k^{p}}\right)=(p+2)(p+d-4)(\boldsymbol{b} \cdot \boldsymbol{k}) \frac{e_{i j}^{-} T^{i j}}{k^{p+2}} \\
& e_{i j}^{-}\left[\frac{b_{k}}{k^{p}} \tilde{K}_{k} T^{i j}-2 p \frac{b_{k}}{k^{p}} \frac{\partial T^{i j}}{\partial k_{k}}+\frac{2}{k^{p}}\left(b_{m} \frac{\partial T^{m j}}{\partial k_{i}}-b^{i} \frac{\partial T^{m j}}{\partial k^{m}}\right)+(i \leftrightarrow j)\right] \\
& +\left(\frac{6 b^{k} e_{k j}^{-} k_{i} T^{i j}}{k^{3}}\right)+(i \leftrightarrow j) .
\end{aligned}
$$

Now, we see that for $p=1$, we have

$$
2 b_{k} \sigma_{\alpha \dot{\alpha}}^{k} \frac{\partial}{\partial \lambda_{\alpha}} \frac{\partial}{\partial \bar{\lambda}_{\dot{\alpha}}}\left(e_{i j}^{-} \frac{T^{i j}}{k}\right)=-b^{k} e_{i j}^{-} K_{k} T^{i j}+\left(\frac{6 b^{k} e_{k j}^{-} k_{i} T^{i j}}{k^{3}}\right)+(i \leftrightarrow j) .
$$

This matches precisely with Eqn. (4.37) of [13], up to the same overall minus sign that appeared above.

The result (C.40) is useful in the following way. Consider a correlation function with some number of T's contracted with polarization tensors. Now, the action of the conformal generator on this object is not very well defined because the polarization tensor is not a well defined function of the momenta. (This is because, given a polarization tensor, we can multiply it by a phase and obtain an equally good tensor.) However, we do know that the conformal generator acting on the bare correlator (without any polarization tensor) vanishes by conformal invariance. What (C.40) tells us is that if we act with the double-derivative operator (which is well-defined on polarization tensors also, as opposed to the original conformal generator) then this is the same as the action of the original conformal generator on the bare correlator (which vanishes) plus a term that is proportional to the Ward identities.

\section{C.5 Relation between $\tilde{R}$ and $S$}

Finally, let us show how spinor identities can be used to derive a relation between $\tilde{R}$ and $S$. Contracting (3.2) with the polarization tensor in (C.13) and using the fact that $e_{i j}^{ \pm} k_{2}^{i}=-e_{i j}^{ \pm} k_{1}^{i}$, which follows $e_{i j}^{ \pm} k_{3}^{i}=-e_{i j}^{ \pm}\left(k_{1}^{i}+k_{2}^{i}\right)=0$, we see that

$$
\begin{aligned}
& \frac{1}{k_{1} k_{2} k_{3}} e^{ \pm, i j}\left\langle O\left(\boldsymbol{k}_{1}\right) O\left(\boldsymbol{k}_{2}\right) T_{i j}\left(\boldsymbol{k}_{3}\right)\right\rangle \\
& =-\frac{1}{k_{1} k_{2} k_{3}} e^{ \pm, i j} k_{1 i} k_{2 j}\left(f_{1}\left(k_{1}, k_{2}, k_{3}\right)+f_{1}\left(k_{2}, k_{1}, k_{3}\right)-2 f_{2}\left(k_{1}, k_{2}, k_{3}\right)\right) \\
& =-\frac{2}{k_{1} k_{2} k_{3}} e^{ \pm, i j} k_{1 i} k_{1 j} S\left(k_{1}, k_{2}, k_{3}\right) .
\end{aligned}
$$

Now, specializing the the negative helicity polarization tensor we can write:

$$
e^{-, i j} k_{1 i} k_{2 j}=\frac{1}{4 k_{3}^{2}}\left\langle\lambda_{3}, \lambda_{1}\right\rangle\left[\lambda_{3}, \bar{\lambda}_{1}\right]\left\langle\lambda_{3}, \lambda_{2}\right\rangle\left[\lambda_{3}, \bar{\lambda}_{2}\right]
$$


We can use some spinor identities to rewrite the amplitude above. These identities simply come from the conservation of momentum, which in the spinor basis, can be written:

$$
\lambda_{1 \alpha} \bar{\lambda}_{1 \dot{\alpha}}+\lambda_{2 \alpha} \bar{\lambda}_{2 \dot{\alpha}}+\lambda_{3 \alpha} \bar{\lambda}_{3 \dot{\alpha}}=\left(k_{1}+k_{2}+k_{3}\right) \sigma_{\alpha \dot{\alpha}}^{0} .
$$

Contracting this with $\lambda_{3}^{\alpha} \bar{\lambda}_{1}^{\dot{\alpha}}$, this leads to:

$$
\left\langle\lambda_{3}, \lambda_{2}\right\rangle\left\langle\bar{\lambda}_{2}, \bar{\lambda}_{1}\right\rangle=-\left(k_{1}+k_{2}+k_{3}\right)\left[\lambda_{3}, \bar{\lambda}_{1}\right],
$$

and we can derive a similar identity

$$
\left\langle\lambda_{3}, \lambda_{1}\right\rangle\left\langle\bar{\lambda}_{1}, \bar{\lambda}_{2}\right\rangle=-\left(k_{1}+k_{2}+k_{3}\right)\left[\lambda_{3}, \bar{\lambda}_{2}\right] .
$$

Moreover, we also have the identity

$$
\begin{aligned}
\left\langle\lambda_{1}, \lambda_{2}\right\rangle\left\langle\bar{\lambda}_{1}, \bar{\lambda}_{2}\right\rangle & =-2\left(\left(\boldsymbol{k}_{\mathbf{1}} \cdot \boldsymbol{k}_{\mathbf{2}}\right)-k_{1} k_{2}\right)=\left(k_{1}+k_{2}\right)^{2}-\left(\boldsymbol{k}_{\mathbf{1}}+\boldsymbol{k}_{\mathbf{2}}\right)^{2} \\
& =\left(k_{1}+k_{2}+k_{3}\right)\left(k_{1}+k_{2}-k_{3}\right) .
\end{aligned}
$$

Putting these relations together we immediately get (3.47).

\section{References}

[1] E. Komatsu, Hunting for Primordial Non-Gaussianity in the Cosmic Microwave Background, Class.Quant.Grav. 27 (2010) p. 124010, [arXiv:1003.6097].

[2] E. Komatsu, N. Afshordi, N. Bartolo, D. Baumann, J. Bond, et. al., Non-Gaussianity as a Probe of the Physics of the Primordial Universe and the Astrophysics of the Low Redshift Universe, arXiv:0902 .4759. ;N. Bartolo, E. Komatsu, S. Matarrese, and A. Riotto, Non-Gaussianity from inflation: Theory and observations, Phys.Rept. 402 (2004) pp. 103-266, [astro-ph/0406398]. ;E. Komatsu, The pursuit of non-gaussian fluctuations in the cosmic microwave background, astro-ph/0206039.

[3] S. Weinberg, Cosmology. Oxford University Press, 2008.

[4] X. Chen, M.-x. Huang, S. Kachru, and G. Shiu, Observational signatures and non-Gaussianities of general single field inflation, JCAP 0701 (2007) p. 002, [hep-th/0605045].

[5] E. Silverstein and D. Tong, Scalar speed limits and cosmology: Acceleration from D-cceleration, Phys.Rev. D70 (2004) p. 103505, [hep-th/0310221]. ;M. Alishahiha, E. Silverstein, and D. Tong, DBI in the sky, Phys.Rev. D70 (2004) p. 123505, [hep-th/0404084].

[6] N. Arkani-Hamed, P. Creminelli, S. Mukohyama, and M. Zaldarriaga, Ghost inflation, JCAP 0404 (2004) p. 001, [hep-th/0312100].

[7] C. Cheung, P. Creminelli, A. L. Fitzpatrick, J. Kaplan, and L. Senatore, The Effective Field Theory of Inflation, JHEP 0803 (2008) p. 14, [arXiv:0709.0293].

[8] H. Osborn and A. Petkou, Implications of conformal invariance in field theories for general dimensions, Annals Phys. 231 (1994) pp. 311-362, [hep-th/9307010]. 
[9] S. Giombi, S. Prakash, and X. Yin, A Note on CFT Correlators in Three Dimensions, arXiv:1104.4317. ;J. Maldacena, A. Zhiboedov, and A. Zhiboedov, Constraining conformal field theories with a slightly broken higher spin symmetry, arXiv:1204.3882. ;J. Maldacena and A. Zhiboedov, Constraining Conformal Field Theories with A Higher Spin Symmetry, arXiv: 1112.1016.

[10] A. Strominger, The dS / CFT correspondence, JHEP 0110 (2001) p. 034, [hep-th/0106113]. ;E. Witten, Quantum gravity in de Sitter space, hep-th/0106109.

[11] J. M. Maldacena, Non-Gaussian features of primordial fluctuations in single field inflationary models, JHEP 0305 (2003) p. 013, [astro-ph/0210603].

[12] D. Anninos, T. Hartman, and A. Strominger, Higher Spin Realization of the $d S / C F T$ Correspondence, arXiv:1108.5735.

[13] J. M. Maldacena and G. L. Pimentel, On graviton non-Gaussianities during inflation, arXiv: 1104.2846.

[14] I. Antoniadis, P. O. Mazur, and E. Mottola, Conformal Invariance, Dark Energy, and CMB Non-Gaussianity, JCAP 1209 (2012) p. 024, [arXiv:1103.4164]. ;A. Bzowski, P. McFadden, and K. Skenderis, Holographic predictions for cosmological 3-point functions, JHEP 1203 (2012) p. 091, [arXiv:1112.1967]. ;P. McFadden and K. Skenderis, Cosmological 3-point correlators from holography, JCAP 1106 (2011) p. 030, [arXiv:1104.3894]. ;P. McFadden and K. Skenderis, Holographic Non-Gaussianity, JCAP 1105 (2011) p. 013, [arXiv:1011.0452]. ;F. Larsen and R. McNees, Inflation and de Sitter holography, JHEP 0307 (2003) p. 051, [hep-th/0307026]. ;F. Larsen, J. P. van der Schaar, and R. G. Leigh, De Sitter holography and the cosmic microwave background, JHEP 0204 (2002) p. 047, [hep-th/0202127].

[15] S. Weinberg, Effective Field Theory for Inflation, Phys.Rev. D77 (2008) p. 123541, [arXiv: 0804.4291].

[16] K. Schalm, G. Shiu, and T. van der Aalst, Consistency condition for inflation from (broken) conformal symmetry, arXiv:1211.2157. ;A. Bzowski, P. McFadden, and K. Skenderis, Holography for inflation using conformal perturbation theory, arXiv:1211.4550.

[17] S. Raju, Four Point Functions of the Stress Tensor and Conserved Currents in $A d S_{4} / C F T_{3}$, Phys.Rev. D85 (2012) p. 126008, [arXiv:1201.6452].

[18] S. Raju, New Recursion Relations and a Flat Space Limit for AdS/CFT Correlators, arXiv:1201.6449.

[19] S. Giombi and X. Yin, The Higher Spin/Vector Model Duality, arXiv:1208.4036.

[20] I. Heemskerk, J. Penedones, J. Polchinski, and J. Sully, Holography from Conformal Field Theory, JHEP 0910 (2009) p. 079, [arXiv:0907.0151].

[21] M. Vasiliev, Higher spin gauge theories in any dimension, Comptes Rendus Physique 5 (2004) pp. 1101-1109, [hep-th/0409260]. 
[22] P. Di Francesco, P. Mathieu, and D. Senechal, Conformal field theory. Springer, New York, USA, 1997.

[23] H. K. Dreiner, H. E. Haber, and S. P. Martin, Two-component spinor techniques and Feynman rules for quantum field theory and supersymmetry, Phys.Rept. 494 (2010) pp. 1-196, [arXiv: 0812.1594]. 